\title{
Assessing the Effect of Including Social Costs in Economic Evaluations of Diabetes-Related Interventions: A Systematic Review
}

\author{
Beatriz Rodriguez-Sanchez (iD) \\ Isaac Aranda-Reneo (iD ${ }^{2}$ \\ Juan Oliva-Moreno iD ${ }^{3}$ \\ Julio Lopez-Bastida ${ }^{4}$ \\ 'University Camilo José Cela, Faculty of \\ Communication and Humanities, Madrid, \\ Spain; ${ }^{2}$ University of Castilla-La Mancha, \\ Faculty of Social Science, Economics and \\ Finance Department, Toledo, Spain; \\ ${ }^{3}$ University of Castilla-La Mancha, Faculty \\ of Law and Social Science, Economics and \\ Finance Department, Toledo, Spain; \\ ${ }^{4}$ University of Castilla-La Mancha, Faculty \\ of Health Sciences, Talavera de la Reina, \\ Toledo, Spain
}

Background: The economic burden of diabetes from a societal perspective is well documented in the cost-of-illness literature. However, the effect of considering social costs in the results and conclusions of economic evaluations of diabetes-related interventions remains unknown.

Objective: To investigate whether the inclusion of social costs (productivity losses and/or informal care) might change the results and conclusions of economic evaluations of diabetesrelated interventions.

Methods: A systematic review was designed and launched on Medline and the CostEffectiveness Analysis Registry from the University of Tufts, from the year 2000 until 2018. Included studies had to fulfil the following criteria: i) being an original study published in a scientific journal, ii) being an economic evaluation of an intervention on diabetes, iii) including social costs, iv) being written in English, v) using quality-adjusted life years as outcome, and vi) separating the results according to the perspective applied.

Results: From the 691 records identified, 47 studies $(6.8 \%)$ were selected. Productivity losses were included in 45 of the selected articles $(73 \%$ used the human capital approach) whereas informal care costs in only 13 (when stated, the opportunity cost method was used in seven studies and the replacement cost in one). The 47 studies resulted in 110 economic evaluation estimations. The inclusion of social costs changed the conclusions in 8 estimations (17\%), 6 of them switching from not cost-effective from the healthcare perspective to cost-effective or dominant from the societal perspective. Considering social costs altered the results from cost-effective to dominant in 9 estimations (19\%).

Conclusion: When social costs are considered, the results and conclusions of economic evaluations performed in diabetes-related interventions can alter. Wide methodological variations have been observed, which limit the comparability of studies and advocate for the inclusion of a wider perspective via the consideration of social costs in economic evaluations and methodological guidelines relating to their estimation and valuation.

Keywords: diabetes, social costs, informal care, productivity losses, economic evaluation, health technology assessment

\section{Plain Language Summary}

- Despite the heavy burden of social costs related to diabetes, there is still a lack of evidence about the consequences of excluding them from economic evaluations of interventions targeting people with diabetes. 
- Our findings showed that, of the 738 records that consisted of a full diabetes-associated economic evaluation, only 106 included social costs $(14.36 \%)$, which differed from those in similar work carried out on other diseases such as Alzheimer's disease and depression, where a higher percentage was found.

- The literature review performed showed that consideration of informal care costs and/or productivity losses changed the results or conclusions in $18 \%$ of economic evaluations of diabetes (in 20 out of 110).

- However, comparisons should be made with caution, as the different types of diabetes affect completely different profiles, with respect to age, state of health and lifestyle.

\section{Introduction}

Diabetes Mellitus (DM) is a disease of high and increasing incidence and prevalence worldwide. In addition to the large number of deaths, loss of health-related quality of life and disability that DM causes directly, it also increases the probability of suffering micro and macrovascular diseases. Thus, its total effect is enormous, and its prevention and the control of related diseases are among the biggest challenges for public health and health systems throughout the world. ${ }^{1,2}$

There is solid evidence of the big economic impact of DM and its associated diseases. ${ }^{3-7}$ The enormous economic and social burdens imposed by DM demand new ways of curbing expenditure on the related healthcare, which includes long-term care. ${ }^{8,9}$ It has been estimated that, in the United States, one in every seven healthcare dollars is spent on treating diabetes and its complications. ${ }^{3,10}$ Additionally, although in the literature there is a predominance of studies that focus on the analysis of the healthcare costs of DM, those studies that have analysed the costs from a broader perspective have identified the relevance of other social costs caused directly by DM (loss of vision, amputation of limbs) or by diseases related to it (ischemic heart disease, stroke, renal failure, etc.). ${ }^{11-13}$ In this sense, diabetes has been found to significantly reduce productivity at work, ${ }^{3,11-13}$ even necessitating an early withdrawal from the labour force, ${ }^{14,15}$ and leading to substantial financial losses among people with diabetes. ${ }^{16,17}$ Moreover, the informal care costs per person with diabetes in the United States have been estimated to range between $\$ 1162$ to $\$ 5082$ per year, which is more than the informal care costs for other diseases, such as heart failure $(\$ 862) .{ }^{13,18}$
In recent years, several studies have tried to analyse the effect of including non-healthcare costs in economic evaluations, adopting a societal perspective instead of the more frequent perspective of the healthcare funder. ${ }^{19-23}$ However, none of these studies included economic evaluations of preventive programmes or treatments related to DM. The general results obtained in the previous studies indicate that (i) non-healthcare costs are only included in a small number of studies, although with significant variations depending on the disease (almost non-existent in rare diseases, ${ }^{24}$ more present in Alzheimer's disease ${ }^{21}$ ); (ii) depending on the disease, the inclusion of non-healthcare costs in the analysis may produce changes in the results, and conclusions that vary from very small to very considerable changes.

The objectives of this research were, first, to verify the presence of economic evaluations that use the societal perspective in the analysis of healthcare-related interventions in the treatment or management of DM. Secondly, to study whether the inclusion of non-healthcare costs (productivity losses and/or informal care costs) can affect the results of economic evaluations and the conclusions reached by their authors.

\section{Methods}

\section{Design}

We designed a systematic review that only included original full economic evaluations of diabetes-related interventions. We used natural language and MesH terms to build a search strategy launched in Medline using PubMed. Although we did not aim to assess which diabetes-related intervention achieved better results, we based our search strategy on the Patient, Intervention, Comparison and Outcome (PICO) framework. This frame has demonstrated a better balance between sensitivity and specificity in identifying references in systematic reviews, reducing the odds of excluding relevant papers. ${ }^{25}$ The following terms were included: ("Costs and Cost Analysis" OR "costeffectiveness" OR “cost-utility" OR "cost-benefit" OR "economic evaluation" OR "economic analysis" OR "QALY" OR "quality-adjusted life years") AND ("Diabetes" OR "Type 2 Diabetes"). We also used diabetes-related terms (such as "diabetes", "diabetes mellitus", "diabetes mellitus type 2") in the Cost-Effectiveness Analysis (CEA) Registry of Tufts University. This registry uses an algorithm, also launched in Medline, and which combines terms such as "cost-effectiveness", "cost- 
utility", "economic evaluation", "QALY" and "qualityadjusted life years". ${ }^{26,27}$ Likewise, we avoided the loss of sensitivity (when identifying economic evaluations carried out in diabetes) which could have resulted if only PubMed had been used. Two researchers carried out the first stage of the review (screening titles and abstracts), and then the same two researchers performed a full-text review of the manuscript included after the review of titles and abstracts. We followed the PRISMA recommendations for carrying out systematic reviews. ${ }^{28}$ All discrepancies were solved by another member of the team who did not participate in the review of titles and abstracts.

\section{Inclusion Criteria}

We included in the review manuscripts that fulfilled the following inclusion criteria: (i) being an original study published in a scientific journal, (ii) being an economic evaluation of any intervention related to diabetes, (iii) including informal care costs and/or productivity losses, which we referred to as social costs, (iv) using qualityadjusted life years as an outcome, (v) separating the results according to the perspective applied (healthcare payer/ provider versus a societal perspective), and (vi) being written in English. Letters to the editor, reports, editorials and brief communications were excluded. The narrative and/or systematic reviews of economic evaluations were also excluded, but we reviewed the economic evaluations included in those reviews to avoid loss of sensitivity in our search strategy through cross-references. We therefore included all economic evaluations carried out on healthcare technologies used as pharmaceutical treatments, medical devices or healthcare programmes for all types of diabetes. Likewise, we included studies carried out on gestational DM, diabetes type 1 or diabetes type 2 .

\section{Data Extraction}

We retrieved the following information from the studies included in the systematic review: the first author's name and year of publication, the cost-effectiveness analysis performed (cost-effectiveness/cost-utility), the country where it was carried out and the currency used, the type of intervention considered in the evaluation, as well as the type of diabetes to which the intervention related. Different available treatments might be identified depending on diabetes-related risk factors (for example, health education programmes to tackle problems related to obesity or glucose measurement patches), the type of diabetes (pharmacological treatment, ie metformin vs insulin), or diabetes-related complications (which range from amputations to blindness and heart attack/stroke). We also extracted the information concerning the threshold for acceptability/recommendation assumed (or stated) by the authors, the discount rates for costs and QALYs, the time horizon, the type of sensitivity analysis performed, the Incremental Cost-Utility Ratios (ICURs) resulting from the cost-effectiveness analysis and, finally, the type of costs included in the analysis. Moreover, given the scope of the systematic review, we recorded information about the method used by the authors, if explicitly identified, to estimate productivity losses and informal care costs, as well as the unit cost applied and the source from which the unit cost was taken.

\section{Data Analysis and Cost Definitions}

Costs and, hence, the costing perspectives considered in this review were defined in accordance with the System of Health Accounts methodology proposed by the Organisation for Economic Co-operation and Development (OECD) in 2000, and revised in 2011, for carrying out economic evaluations of healthcare programmes. ${ }^{29}$ More precisely, the set of healthcare costs would encompass the more traditional items, such as visits to general practitioners (GP) and specialists, outpatient consultations, pharmaceuticals, hospitalisations, imaging and laboratory tests, emergency visits; but also nursing homes, community-based care, social services, out-ofpocket healthcare expenditure and the cost of travelling to be treated. According to the revised edition of the report launched by the OECD in 2011,

total long-term care consists of a range of medical/nursing care services, personal care services and assistance services that are consumed with the primary goal of alleviating pain and suffering or reducing or managing the deterioration in health status in patients with a degree of long-term dependency.

Hence, the aforementioned costs (healthcare costs in addition to the costs of social services) entailed the "healthcare payer/provider perspective" throughout the current review. On the other hand, a "societal perspective" referred to the scenario in which, additionally to the previous costs, productivity losses, defined as the economic valuation of paid worktime lost by the patients as a result of the disease, and informal care costs, defined as the economic valuation of the time allotted to care activities by a non-professional caregiver (friend or relative), were included. It should be 
noted that the societal perspective should be applied not only to the costs, but also to the effects on the health of both the people who suffer from the disease and the members of their environment (relatives, friends). However, these effects are hardly ever included, and the consideration of a societal perspective is usually limited to costs.

We recorded a change in the conclusions when the decision about the adoption of a new technology was modified as a result of the inclusion of the social costs. For instance, from the healthcare perspective, the ICUR was above the threshold value, so the assessed technology was not recommended. But when social costs were included, the ICUR was modified so that it lay below the corresponding threshold. On the other hand, a change in results was identified when social costs were introduced and (i) ICUR fell below the threshold (the previous case referred to); (ii) the intervention became cost-saving (although it was previously already cost effective, but had a positive ICUR). It is important to stress that a significant change in the results may not change the conclusions of the analysis. For example, an intervention assessed with a favourable ICUR from the healthcare perspective would be recommended. If the inclusion of social costs made the ratio significantly more favourable (or even dominant), there would have been a change in the result of the economic evaluation, but the recommendation would not have been altered, as the evaluated intervention would have already been recommended from the healthcare perspective.

\section{Results}

The search strategy launched in PubMed retrieved 7421 potential references to be included in the review. In the CEA registry, we retrieved nearly 500 references. After removing the duplicates, we screened 7857 records obtained from PubMed and the CEA registry. Figure 1 includes the PRISMA flowchart explaining the numbers at each stage of the systematic review. Briefly, we found 691 economic evaluations of healthcare-related technologies, of which only $47(6.8 \%)$ fulfilled all the inclusion criteria. The main reason for exclusion was not including social costs (Figure 1, $\mathrm{n}=632$; 91.5\%). 31 additional manuscripts considered social costs, but either it was not possible to extract the ICURs from both perspectives or the costs analysis was not reported separately. Finally, 28 studies did not include QALYs as an outcome.

\section{Description of the Studies Included}

The majority of the studies included in the review were carried out in Europe $(\mathrm{n}=31,66 \%) .{ }^{30-60}$ In North America, 12 additional studies were carried out $(25.5 \%)^{61-72}$ and only 4 studies took place in Asian countries $(n=4$; $8.5 \%) .{ }^{73-76} \mathrm{~A}$ pharmaceutical intervention was the diabetes-related intervention being assessed in the largest number of studies $\quad(\mathrm{n}=18$; $38.3 \%), 30,33,35-38,41,42,48-53,56,57,59-61,71-73$ followed by healthcare educational or behavioural programmes (including physical advisory and nutritional programmes). ${ }^{31,32,34,39,43,45-47,54,63,65,67,76}$ Only one study assessed a surgical intervention aimed at treating painful diabetic peripheral neuropathy using Spinal Cord Stimulation (SCS). ${ }^{55}$ With respect to the time horizon of the economic evaluations performed, lifetime was the most prevalent one, as it was considered in 21 studies (45\%). ${ }^{30,31,37,40-42,44,48-53,56,57,59,66-68,71,75}$ Shorter time horizons (of one year or less) were found in 10 of the 47 studies included in the review. ${ }^{32-34,36,39,46,47,55,58,73}$

Regarding the social costs included in the studies, informal care costs were included in 13 studies, ${ }^{34,39,43,46,47,54,55,61,62,64,66,68,71}$ whereas the economic evaluation of productivity losses was included in most of them $(n=45)$ (see Table 1). Moreover, of the 13 studies that considered informal care costs, the authors of 11 of them additionally included productivity losses, both these social costs being considered in this review together. ${ }^{34,39,43,46,47,54,55,61,66,68,71}$ Hence, informal care costs were only included in 2 studies, ${ }^{62,64}$ whereas productivity losses costs were individually included in 34 references. So, productivity losses were included much more frequently than informal care costs in the economic evaluation of diabetes-related healthcare interventions.

The method most frequently used to estimate productivity losses was the human capital approach. The friction cost method was applied in five studies, ${ }^{31,34,46,55,58}$ but only two studies explicitly indicated the length of the friction period. de Wit et $\mathrm{al}^{34}$ used 84 days and Oostdam et al. ${ }^{46} 154$ calendar days and an elasticity correction factor of 0.8 . Moreover, five studies included not only the loss of production due to absenteeism but also those losses attributable to presenteeism. ${ }^{33,34,47,63,68}$ de Salas-Cansado et al ${ }^{33}$ directly asked the participants in the study about the patient's selfperceived average productivity at work (determined as $0-100 \%$ productivity). The authors then applied a formula to estimate the number of Lost Workday Equivalents. On the 


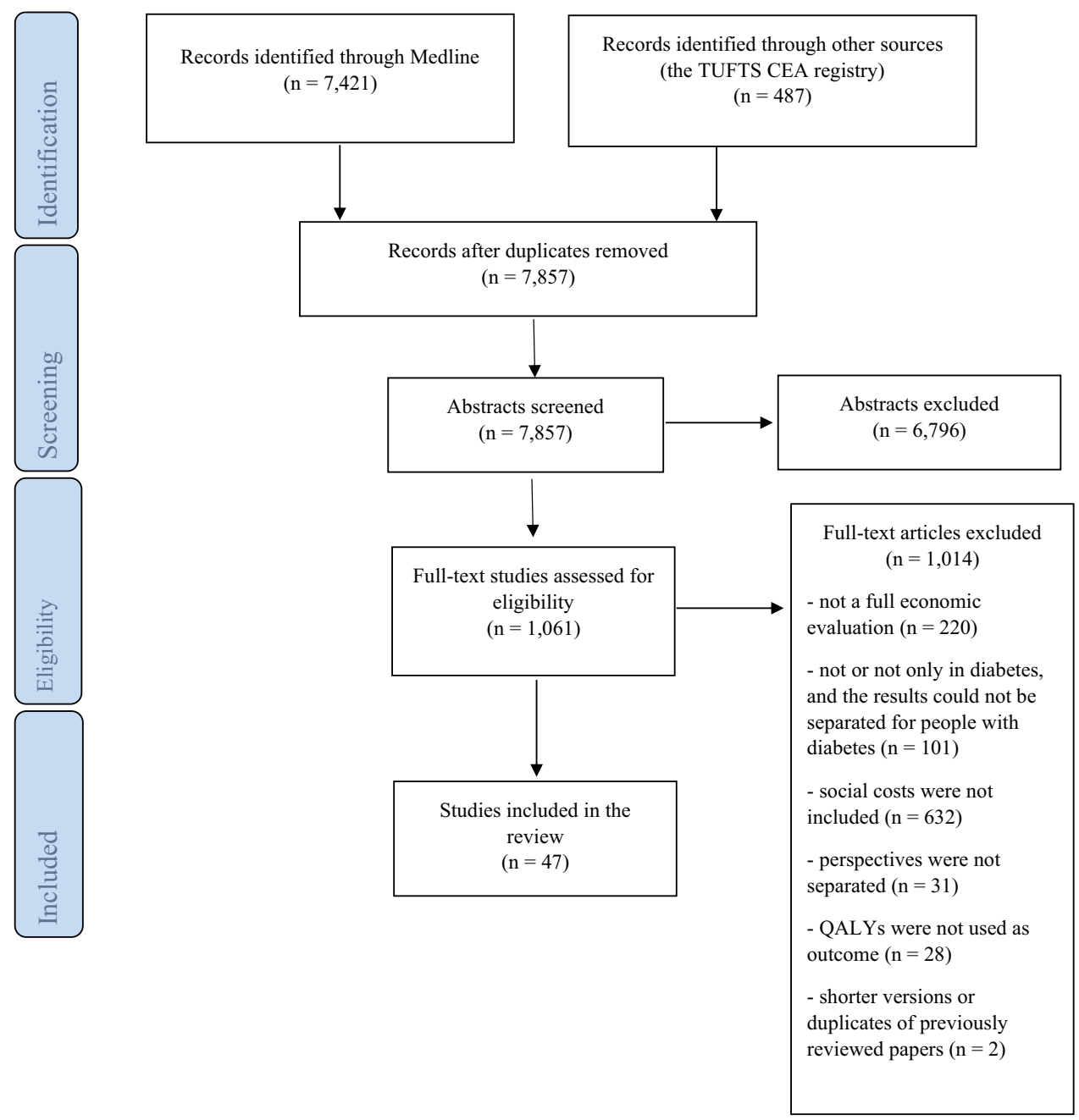

Figure I PRISMA flowchart of the search strategy.

other hand, Eddy et $\mathrm{al}^{63}$ used the Quality of Well-being Index to estimate decreased productivity at work. In four of the articles the authors did not state the method applied for assessing productivity losses. ${ }^{48,66,71,73}$

The informal care costs were estimated mainly by using the opportunity cost method $(n=6),{ }^{39,43,54,55,62,68}$ and only one study also used the good proxy method to assess the time of informal care. ${ }^{54}$ The method used to estimate informal care costs was not reported in seven studies. ${ }^{34,46,47,61,64,66,71}$ Table 1 provides a further detailed description of the studies included in the review.

\section{Effect of Including Social Costs on the Results and Conclusions of the Economic Evaluations Performed}

From the 47 studies included in the review, it was possible to obtain 110 cost-utility analyses/estimations, as one study could perform more than one economic evaluation, as was the case of de Wit et $\mathrm{al}^{34}$ and Haig et al. ${ }^{66}$ All these estimations are provided in Table A1 with more detail from the Appendix. Table 2 shows the twenty ICUR estimations (18.2\%) from seventeen studies where we observed changes in results or authors' conclusions after consideration of the social costs.

The conclusions of the economic evaluation changed in 8 estimations from 7 studies $^{32,40,51,52,55,62,63}$ when the societal perspective was considered. The inclusion of social costs in most cases (estimations number 18, 37, 41,44 and 104) led to the intervention becoming costeffective. Hence, the ICUR from the assessed intervention was above the threshold value from the healthcare payer's perspective and, once social costs were considered, the ICUR fell below the corresponding threshold. However, there were 2 additional estimations where the opposite change in conclusions was observed (estimations numbers 
Table I Descriptive Information on the Selected Studies $(n=47)$

\begin{tabular}{|c|c|c|c|c|c|c|c|}
\hline $\begin{array}{l}\text { First Author \& } \\
\text { Publication } \\
\text { Year }\end{array}$ & $\begin{array}{c}\text { Diabetes } \\
\text { Type }\end{array}$ & $\begin{array}{c}\text { Intervention } \\
\text { Type }\end{array}$ & $\begin{array}{l}\text { Country } \\
\text { and } \\
\text { Currency }\end{array}$ & $\begin{array}{c}\text { Discount } \\
\text { Rate } \\
\text { (Costs/ } \\
\text { Outcomes) }\end{array}$ & $\begin{array}{c}\text { Time } \\
\text { Horizon }\end{array}$ & Costs Included & $\begin{array}{l}\text { Method Used } \\
\text { for Calculating } \\
\text { Social Costs }\end{array}$ \\
\hline $\begin{array}{l}\text { Broekhuizen } \\
(2018)^{32}\end{array}$ & $\begin{array}{c}\text { Gestational } \\
\text { diabetes } \\
\text { mellitus }\end{array}$ & $\begin{array}{c}\text { Health education } \\
\text { or behaviour }\end{array}$ & $\begin{array}{l}\text { United } \\
\text { Kingdom, } \\
\text { Ireland, } \\
\text { Austria, } \\
\text { Poland, Italy, } \\
\text { Spain, } \\
\text { Denmark, } \\
\text { Belgium, and } \\
\text { the } \\
\text { Netherlands } \\
(2012 €)\end{array}$ & n.a./n.a. & 48 weeks & $\begin{array}{l}\text { Healthcare costs: } \\
\text { primary and } \\
\text { secondary } \\
\text { healthcare, } \\
\text { medication, travel } \\
\text { costs } \\
\text { Social costs: } \\
\text { productivity losses }\end{array}$ & $\begin{array}{l}\text { Productivity } \\
\text { losses: human } \\
\text { capital approach } \\
\text { (paid time - } \\
\text { absenteeism) }\end{array}$ \\
\hline De Wit $(2018)^{34}$ & $\begin{array}{l}\text { Diabetes } \\
\text { mellitus } \\
\text { type I or } \\
\text { type } 2\end{array}$ & $\begin{array}{c}\text { Health education } \\
\text { or behaviour }\end{array}$ & $\begin{array}{c}\text { The } \\
\text { Netherlands } \\
(2015 €)\end{array}$ & n.a/n.a. & 6 months & $\begin{array}{l}\text { Healthcare costs: } \\
\text { hospital admissions, } \\
\text { outpatient visits and } \\
\text { calls, emergency } \\
\text { room visits, } \\
\text { ambulance transfers, } \\
\text { medication and } \\
\text { medical supply usage } \\
\text { Social costs: } \\
\text { productivity losses } \\
\text { and informal care }\end{array}$ & $\begin{array}{c}\text { Productivity } \\
\text { losses: friction } \\
\text { cost method } \\
\text { (paid - } \\
\text { absenteeism and } \\
\text { presenteeism - } \\
\text { and unpaid } \\
\text { work) } \\
\text { Informal care: } \mathrm{n} \text {. } \\
\text { a. }\end{array}$ \\
\hline Ericsson $(2018)^{35}$ & $\begin{array}{l}\text { Diabetes } \\
\text { mellitus } \\
\text { type } 2\end{array}$ & Pharmaceutical & $\begin{array}{l}\text { Sweden } \\
\text { (20I5SEKs) }\end{array}$ & $3 \% ; 3 \%$ & 40 years & $\begin{array}{l}\text { Healthcare costs: } \\
\text { anti-hyperglycaemic } \\
\text { treatment and other } \\
\text { treatment costs, } \\
\text { diabetes-related } \\
\text { complications } \\
\text { Social costs: } \\
\text { productivity losses }\end{array}$ & $\begin{array}{l}\text { Productivity } \\
\text { losses: human } \\
\text { capital approach } \\
\text { (paid time - } \\
\text { absenteeism) }\end{array}$ \\
\hline Breeze $(2017)^{31}$ & Diabetes & $\begin{array}{c}\text { Health education } \\
\text { or behaviour }\end{array}$ & $\begin{array}{c}\text { UK }(20 \mid 4 / 15 \\
£)\end{array}$ & $1.5 \%, 1.5 \%$ & Lifetime & $\begin{array}{l}\text { Healthcare costs: } \\
\text { direct health care } \\
\text { costs, intervention } \\
\text { and HbAlc testing } \\
\text { costs } \\
\text { Social costs: } \\
\text { productivity losses }\end{array}$ & $\begin{array}{l}\text { Productivity } \\
\text { losses: friction } \\
\text { cost approach } \\
\text { (paid time - } \\
\text { absenteeism) }\end{array}$ \\
\hline Jendel $(2017)^{40}$ & $\begin{array}{l}\text { Diabetes } \\
\text { mellitus } \\
\text { type I }\end{array}$ & $\begin{array}{l}\text { Medical device/ } \\
\text { pharmaceutical }\end{array}$ & $\begin{array}{l}\text { Sweden } \\
\text { (20I5SEKs) }\end{array}$ & $3 \% ; 3 \%$ & Lifetime & $\begin{array}{l}\text { Healthcare costs: } \\
\text { intervention costs } \\
\text { (insulin sensor), self- } \\
\text { monitoring of blood } \\
\text { glucose strips, } \\
\text { diabetes-related } \\
\text { complications } \\
\text { Social costs: } \\
\text { productivity losses }\end{array}$ & $\begin{array}{l}\text { Productivity } \\
\text { losses: human } \\
\text { capital approach } \\
\text { (paid time - } \\
\text { absenteeism) }\end{array}$ \\
\hline
\end{tabular}

(Continued) 
Table I (Continued).

\begin{tabular}{|c|c|c|c|c|c|c|c|}
\hline $\begin{array}{l}\text { First Author \& } \\
\text { Publication } \\
\text { Year }\end{array}$ & $\begin{array}{c}\text { Diabetes } \\
\text { Type }\end{array}$ & $\begin{array}{c}\text { Intervention } \\
\text { Type }\end{array}$ & $\begin{array}{l}\text { Country } \\
\text { and } \\
\text { Currency }\end{array}$ & $\begin{array}{l}\text { Discount } \\
\text { Rate } \\
\text { (Costs/ } \\
\text { Outcomes) }\end{array}$ & $\begin{array}{c}\text { Time } \\
\text { Horizon }\end{array}$ & Costs Included & $\begin{array}{l}\text { Method Used } \\
\text { for Calculating } \\
\text { Social Costs }\end{array}$ \\
\hline $\begin{array}{l}\text { Landstedt- } \\
\text { Hallin }(2017)^{44}\end{array}$ & $\begin{array}{l}\text { Diabetes } \\
\text { mellitus } \\
\text { type I }\end{array}$ & $\begin{array}{l}\text { Medical device/ } \\
\text { pharmaceutical }\end{array}$ & $\begin{array}{c}\text { Sweden } \\
\text { (20I5SEKs) }\end{array}$ & $3 \% ; 3 \%$ & Lifetime & $\begin{array}{l}\text { Healthcare costs: } \\
\text { direct health care } \\
\text { costs financed by tax } \\
\text { payments and co- } \\
\text { payments } \\
\text { Social costs: } \\
\text { productivity losses }\end{array}$ & $\begin{array}{l}\text { Productivity } \\
\text { losses: human } \\
\text { capital approach } \\
\text { (paid time - } \\
\text { absenteeism) }\end{array}$ \\
\hline Roze $(2017)^{49}$ & $\begin{array}{l}\text { Diabetes } \\
\text { mellitus } \\
\text { type I }\end{array}$ & Medical device & $\begin{array}{c}\text { Denmark } \\
\text { (20I5DKK) }\end{array}$ & $3 \% ; 3 \%$ & Lifetime & $\begin{array}{l}\text { Healthcare costs: } \\
\text { direct medical costs } \\
\text { due to diabetes- } \\
\text { related } \\
\text { complications, } \\
\text { intervention costs } \\
\text { (blood glucose self- } \\
\text { testing) } \\
\text { Social costs: } \\
\text { productivity losses }\end{array}$ & $\begin{array}{c}\text { Productivity } \\
\text { losses: human } \\
\text { capital approach } \\
\text { (paid time - } \\
\text { absenteeism) }\end{array}$ \\
\hline Slangen $(2017)^{55}$ & $\begin{array}{c}\text { Diabetes } \\
\text { mellitus } \\
\text { type } 2\end{array}$ & Surgical & $\begin{array}{c}\text { Netherlands } \\
(2012 €)\end{array}$ & $4 \% ; 1.5 \%$ & $\begin{array}{c}12 \\
\text { months }\end{array}$ & $\begin{array}{l}\text { Healthcare costs: } \\
\text { specialist doctor, } \\
\text { surgery, laboratory } \\
\text { tests, revisions, } \\
\text { paramedical visits, } \\
\text { follow-up visits, } \\
\text { medication, inpatient } \\
\text { admission costs } \\
\text { Social costs: } \\
\text { productivity losses } \\
\text { and informal care } \\
\text { costs }\end{array}$ & $\begin{array}{l}\text { Productivity } \\
\text { losses: friction } \\
\text { cost method } \\
\text { (paid time - } \\
\text { absenteeism) } \\
\text { Informal care: } \\
\text { opportunity cost } \\
\text { method }\end{array}$ \\
\hline Farshchi $(2016)^{73}$ & $\begin{array}{c}\text { Diabetes } \\
\text { mellitus } \\
\text { type } 2\end{array}$ & Pharmaceutical & $\begin{array}{c}\text { Iran }(2012 \text { US } \\
\$)\end{array}$ & n.a/n.a. & 48 weeks & $\begin{array}{l}\text { Healthcare costs: } \\
\text { laboratory, } \\
\text { medications, clinician } \\
\text { visits, inpatient, non- } \\
\text { medical costs } \\
\text { Social costs: } \\
\text { productivity losses }\end{array}$ & $\begin{array}{l}\text { Productivity } \\
\text { losses: n.a. }\end{array}$ \\
\hline Haig $(2016)^{66}$ & $\begin{array}{l}\text { Diabetes } \\
\text { mellitus } \\
\text { type I or } \\
\text { type } 2\end{array}$ & $\begin{array}{c}\text { Medical } \\
\text { procedure, } \\
\text { pharmaceutical }\end{array}$ & $\begin{array}{c}\text { Canada } \\
(2013 \mathrm{CAN} \$)\end{array}$ & $5 \% ; 5 \%$ & Lifetime & $\begin{array}{l}\text { Healthcare costs: } \\
\text { treatment and } \\
\text { monitoring visits, } \\
\text { complications, } \\
\text { treatment costs } \\
\text { Social costs: } \\
\text { productivity losses } \\
\text { and informal care } \\
\text { costs }\end{array}$ & $\begin{array}{l}\text { Productivity } \\
\text { losses: } n . a . \\
\text { Informal care: } \mathrm{n} \text {. } \\
\text { a. }\end{array}$ \\
\hline
\end{tabular}

(Continued) 
Table I (Continued).

\begin{tabular}{|c|c|c|c|c|c|c|c|}
\hline $\begin{array}{l}\text { First Author \& } \\
\text { Publication } \\
\text { Year }\end{array}$ & $\begin{array}{c}\text { Diabetes } \\
\text { Type }\end{array}$ & $\begin{array}{c}\text { Intervention } \\
\text { Type }\end{array}$ & $\begin{array}{l}\text { Country } \\
\text { and } \\
\text { Currency }\end{array}$ & $\begin{array}{c}\text { Discount } \\
\text { Rate } \\
\text { (Costs/ } \\
\text { Outcomes) }\end{array}$ & $\begin{array}{c}\text { Time } \\
\text { Horizon }\end{array}$ & Costs Included & $\begin{array}{l}\text { Method Used } \\
\text { for Calculating } \\
\text { Social Costs }\end{array}$ \\
\hline Kolu $(2016)^{43}$ & $\begin{array}{l}\text { Diabetes } \\
\text { mellitus } \\
\text { type } 2\end{array}$ & $\begin{array}{c}\text { Health education } \\
\text { intervention }\end{array}$ & $\begin{array}{c}\text { Finland (2015 } \\
€)\end{array}$ & Not specified & 7 years & $\begin{array}{l}\text { Healthcare costs: } \\
\text { primary care doctor, } \\
\text { specialist doctor, } \\
\text { nursing, } \\
\text { physiotherapist, } \\
\text { medication, inpatient } \\
\text { admission costs } \\
\text { Social costs: } \\
\text { productivity losses } \\
\text { and informal care } \\
\text { costs }\end{array}$ & $\begin{array}{l}\text { Productivity } \\
\text { losses: human } \\
\text { capital approach } \\
\text { (paid time - } \\
\text { absenteeism } \\
\text { costs) } \\
\text { Informal care: } \\
\text { opportunity cost } \\
\text { method (paid } \\
\text { time) }\end{array}$ \\
\hline $\operatorname{Lian}(2016)^{74}$ & Diabetes & Screening & $\begin{array}{c}\text { China } \\
(2009 \mathrm{HK} \$)\end{array}$ & $3.5 \% ; 3.5 \%$ & n.a. & $\begin{array}{l}\text { Healthcare costs: } \\
\text { staff time costs, co- } \\
\text { payment intervention } \\
\text { cost, capital costs, } \\
\text { follow-up and } \\
\text { treatment costs } \\
\text { Social costs: } \\
\text { productivity losses }\end{array}$ & $\begin{array}{l}\text { Productivity } \\
\text { losses: human } \\
\text { capital approach } \\
\text { (paid time - } \\
\text { absenteeism) }\end{array}$ \\
\hline Nguyen $(2016)^{75}$ & $\begin{array}{l}\text { Diabetes } \\
\text { mellitus } \\
\text { type } 2\end{array}$ & Screening & $\begin{array}{l}\text { Singapore } \\
(2015 \\
\text { Singapore } \$)\end{array}$ & $3 \% ; 3 \%$ & Lifetime & $\begin{array}{l}\text { Healthcare costs: } \\
\text { screening, follow-up } \\
\text { visits, laser } \\
\text { treatment, } \\
\text { transportation costs } \\
\text { Social costs: } \\
\text { productivity losses }\end{array}$ & $\begin{array}{l}\text { Productivity } \\
\text { losses: human } \\
\text { capital approach } \\
\text { (paid time - } \\
\text { absenteeism) }\end{array}$ \\
\hline Roussel $(2016)^{48}$ & $\begin{array}{l}\text { Diabetes } \\
\text { mellitus } \\
\text { type } 2\end{array}$ & Pharmaceutical & $\begin{array}{c}\text { France }(2013 \\
€)\end{array}$ & $3 \% ; 3 \%$ & Lifetime & $\begin{array}{l}\text { Healthcare costs: } \\
\text { diabetes medications, } \\
\text { self-monitoring of } \\
\text { blood glucose, } \\
\text { concomitant } \\
\text { medications and } \\
\text { diabetes-related } \\
\text { complications costs } \\
\text { Social costs: } \\
\text { productivity losses }\end{array}$ & $\begin{array}{c}\text { Productivity } \\
\text { losses: n.a. (paid } \\
\text { time - } \\
\text { absenteeism) }\end{array}$ \\
\hline Roze $(2016)^{53}$ & $\begin{array}{l}\text { Diabetes } \\
\text { mellitus } \\
\text { type I }\end{array}$ & $\begin{array}{l}\text { Medical device, } \\
\text { pharmaceutical }\end{array}$ & $\begin{array}{l}\text { United } \\
\text { Kingdom } \\
(2013 t)\end{array}$ & $3.5 \% ; 1.5 \%$ & Lifetime & $\begin{array}{l}\text { Healthcare costs: } \\
\text { treatment costs } \\
\text { (strips, lancets, } \\
\text { transmitter and } \\
\text { glucose sensors) and } \\
\text { diabetes-related } \\
\text { complication costs } \\
\text { Social costs: } \\
\text { productivity losses }\end{array}$ & $\begin{array}{l}\text { Productivity } \\
\text { losses: human } \\
\text { capital approach } \\
\text { (paid time - } \\
\text { absenteeism) }\end{array}$ \\
\hline
\end{tabular}

(Continued) 
Table I (Continued).

\begin{tabular}{|c|c|c|c|c|c|c|c|}
\hline $\begin{array}{l}\text { First Author \& } \\
\text { Publication } \\
\text { Year }\end{array}$ & $\begin{array}{c}\text { Diabetes } \\
\text { Type }\end{array}$ & $\begin{array}{c}\text { Intervention } \\
\text { Type }\end{array}$ & $\begin{array}{l}\text { Country } \\
\text { and } \\
\text { Currency }\end{array}$ & $\begin{array}{c}\text { Discount } \\
\text { Rate } \\
\text { (Costs/ } \\
\text { Outcomes) }\end{array}$ & $\begin{array}{c}\text { Time } \\
\text { Horizon }\end{array}$ & Costs Included & $\begin{array}{l}\text { Method Used } \\
\text { for Calculating } \\
\text { Social Costs }\end{array}$ \\
\hline Roze $(2016)^{52}$ & $\begin{array}{l}\text { Diabetes } \\
\text { mellitus } \\
\text { type I }\end{array}$ & $\begin{array}{l}\text { Medical device, } \\
\text { pharmaceutical }\end{array}$ & $\begin{array}{c}\text { France }(2014 \\
€)\end{array}$ & $4 \% ; 4 \%$ & Lifetime & $\begin{array}{l}\text { Healthcare costs: } \\
\text { treatment costs } \\
\text { (strips, lancets, } \\
\text { transmitter and } \\
\text { glucose sensors) and } \\
\text { diabetes-related } \\
\text { complication costs } \\
\text { Social costs: } \\
\text { productivity losses }\end{array}$ & $\begin{array}{l}\text { Productivity } \\
\text { losses: human } \\
\text { capital approach } \\
\text { (paid time - } \\
\text { absenteeism) }\end{array}$ \\
\hline Roze $(2016)^{50}$ & $\begin{array}{l}\text { Diabetes } \\
\text { mellitus } \\
\text { type I }\end{array}$ & Pharmaceutical & $\begin{array}{c}\text { Netherlands } \\
(2013 €)\end{array}$ & $4 \% ; 1.5 \%$ & Lifetime & $\begin{array}{l}\text { Healthcare costs: } \\
\text { treatment costs } \\
\text { (strips, lancets, } \\
\text { transmitter and } \\
\text { glucose sensors) and } \\
\text { diabetes-related } \\
\text { complication costs } \\
\text { Social costs: } \\
\text { productivity losses }\end{array}$ & $\begin{array}{l}\text { Productivity } \\
\text { losses: human } \\
\text { capital approach } \\
\text { (paid time - } \\
\text { absenteeism) }\end{array}$ \\
\hline Brown $(2015)^{61}$ & $\begin{array}{l}\text { Diabetes } \\
\text { mellitus } \\
\text { type I or } \\
\text { type } 2\end{array}$ & Pharmaceutical & US (20I2US\$) & $3 \% ; 3 \%$ & 14 years & $\begin{array}{l}\text { Healthcare costs: } \\
\text { disease management } \\
\text { and treatment costs, } \\
\text { complications and } \\
\text { adverse events costs, } \\
\text { insurer costs } \\
\text { Social costs: } \\
\text { productivity losses } \\
\text { and informal care } \\
\text { costs }\end{array}$ & $\begin{array}{l}\text { Productivity } \\
\text { losses: human } \\
\text { capital approach } \\
\text { (paid time) }\end{array}$ \\
\hline Cutino $(2015)^{62}$ & $\begin{array}{l}\text { Diabetes } \\
\text { mellitus } \\
\text { type I or } \\
\text { type } 2\end{array}$ & Medical device & US (20I4US\$) & Not specified & 15 years & $\begin{array}{l}\text { Healthcare costs: } \\
\text { study drug costs, } \\
\text { administration and } \\
\text { monitoring costs, } \\
\text { concomitant } \\
\text { treatments, adverse } \\
\text { events } \\
\text { Social costs: informal } \\
\text { care costs }\end{array}$ & $\begin{array}{l}\text { Informal care: } \\
\text { opportunity } \\
\text { costs (paid time) }\end{array}$ \\
\hline Huetson $(2015)^{38}$ & $\begin{array}{l}\text { Diabetes } \\
\text { mellitus } \\
\text { type } 2\end{array}$ & Pharmaceutical & $\begin{array}{c}\text { Norway } \\
\text { (20I2NOKs) }\end{array}$ & $4 \% ; 4 \%$ & 45 years & $\begin{array}{l}\text { Healthcare costs: } \\
\text { disease management } \\
\text { and treatment costs, } \\
\text { complications costs } \\
\text { Social costs: } \\
\text { productivity losses }\end{array}$ & $\begin{array}{l}\text { Productivity } \\
\text { losses: human } \\
\text { capital approach } \\
\text { (paid time) }\end{array}$ \\
\hline
\end{tabular}

(Continued) 
Table I (Continued).

\begin{tabular}{|c|c|c|c|c|c|c|c|}
\hline $\begin{array}{l}\text { First Author \& } \\
\text { Publication } \\
\text { Year }\end{array}$ & $\begin{array}{c}\text { Diabetes } \\
\text { Type }\end{array}$ & $\begin{array}{c}\text { Intervention } \\
\text { Type }\end{array}$ & $\begin{array}{l}\text { Country } \\
\text { and } \\
\text { Currency }\end{array}$ & $\begin{array}{l}\text { Discount } \\
\text { Rate } \\
\text { (Costs/ } \\
\text { Outcomes) }\end{array}$ & $\begin{array}{c}\text { Time } \\
\text { Horizon }\end{array}$ & Costs Included & $\begin{array}{l}\text { Method Used } \\
\text { for Calculating } \\
\text { Social Costs }\end{array}$ \\
\hline Roze $(2015)^{51}$ & $\begin{array}{l}\text { Diabetes } \\
\text { mellitus } \\
\text { type I }\end{array}$ & Medical device & $\begin{array}{c}\text { Sweden } \\
\text { (20IISEKs) }\end{array}$ & $3 \% ; 3 \%$ & Lifetime & $\begin{array}{l}\text { Healthcare costs: } \\
\text { intervention costs } \\
\text { and diabetes-related } \\
\text { complications costs } \\
\text { (cardiovascular, } \\
\text { renal, acute events, } \\
\text { eye disease and } \\
\text { other) } \\
\text { Social costs: } \\
\text { productivity losses }\end{array}$ & $\begin{array}{l}\text { Productivity } \\
\text { losses: human } \\
\text { capital approach } \\
\text { (paid time - } \\
\text { absenteeism) }\end{array}$ \\
\hline Kiadaliri $(2014)^{42}$ & $\begin{array}{l}\text { Diabetes } \\
\text { mellitus } \\
\text { type } 2\end{array}$ & Pharmaceutical & $\begin{array}{c}\text { Sweden } \\
(20 \text { I4SEKs) }\end{array}$ & n.a/n.a. & Lifetime & $\begin{array}{l}\text { Healthcare costs: } \\
\text { drugs, self- } \\
\text { monitoring blood } \\
\text { glucose test strips } \\
\text { and lancets, diabetes- } \\
\text { related complications } \\
\text { costs, treatment side } \\
\text { effects costs } \\
\text { Social costs: } \\
\text { productivity losses }\end{array}$ & $\begin{array}{c}\text { Productivity } \\
\text { losses: human } \\
\text { capital approach }\end{array}$ \\
\hline Png $(2014)^{76}$ & $\begin{array}{l}\text { Diabetes } \\
\text { mellitus } \\
\text { type } 2 \text { and } \\
\text { prediabetes }\end{array}$ & $\begin{array}{l}\text { Health education } \\
\text { or behaviour - } \\
\text { Pharmaceutical }\end{array}$ & $\begin{array}{l}\text { Singapore } \\
(2012 U S \$)\end{array}$ & $3 \% ; 3 \%$ & 3 years & $\begin{array}{l}\text { Healthcare costs: } \\
\text { outpatient care, } \\
\text { laboratory tests and } \\
\text { medications } \\
\text { Social costs: } \\
\text { productivity losses }\end{array}$ & $\begin{array}{l}\text { Productivity } \\
\text { losses: human } \\
\text { capital approach } \\
\text { (paid time - } \\
\text { absenteeism) }\end{array}$ \\
\hline $\begin{array}{l}\text { Steen-Carlsson \& } \\
\text { Persson }(2014)^{57}\end{array}$ & $\begin{array}{l}\text { Diabetes } \\
\text { mellitus } \\
\text { type } 2\end{array}$ & Pharmaceutical & $\begin{array}{c}\text { Sweden } \\
\text { (20I3SEKs) }\end{array}$ & n.a/n.a. & Lifetime & $\begin{array}{l}\text { Healthcare costs: } \\
\text { preventive } \\
\text { treatment, micro- } \\
\text { and macrovascular } \\
\text { complications costs } \\
\text { Social costs: } \\
\text { productivity losses }\end{array}$ & $\begin{array}{c}\text { Productivity } \\
\text { losses: human } \\
\text { capital approach }\end{array}$ \\
\hline
\end{tabular}

(Continued) 
Table I (Continued).

\begin{tabular}{|c|c|c|c|c|c|c|c|}
\hline $\begin{array}{l}\text { First Author \& } \\
\text { Publication } \\
\text { Year }\end{array}$ & $\begin{array}{c}\text { Diabetes } \\
\text { Type }\end{array}$ & $\begin{array}{c}\text { Intervention } \\
\text { Type }\end{array}$ & $\begin{array}{l}\text { Country } \\
\text { and } \\
\text { Currency }\end{array}$ & $\begin{array}{l}\text { Discount } \\
\text { Rate } \\
\text { (Costs/ } \\
\text { Outcomes) }\end{array}$ & $\begin{array}{c}\text { Time } \\
\text { Horizon }\end{array}$ & Costs Included & $\begin{array}{l}\text { Method Used } \\
\text { for Calculating } \\
\text { Social Costs }\end{array}$ \\
\hline $\begin{array}{l}\text { Tsiachristas } \\
(2014)^{58}\end{array}$ & $\begin{array}{l}\text { Diabetes } \\
\text { mellitus } \\
\text { type } 2\end{array}$ & $\begin{array}{l}\text { Management } \\
\text { program } \\
\text { intervention }\end{array}$ & $\begin{array}{c}\text { Netherlands } \\
(2012 €)\end{array}$ & n.a/n.a. & $\begin{array}{c}12 \\
\text { months }\end{array}$ & $\begin{array}{l}\text { Healthcare costs: GP, } \\
\text { nurse practitioner, } \\
\text { nurse, dietician, } \\
\text { physiotherapist, } \\
\text { podiatrist, lifestyle } \\
\text { coach, medical } \\
\text { specialists in } \\
\text { outpatient clinics } \\
\text { etc., hospital } \\
\text { admissions and } \\
\text { admission days, and } \\
\text { medication use } \\
\text { Social costs: } \\
\text { productivity losses }\end{array}$ & $\begin{array}{l}\text { Productivity } \\
\text { losses: friction } \\
\text { cost method } \\
\text { (paid time - } \\
\text { absenteeism) }\end{array}$ \\
\hline Ericsson $(2013)^{36}$ & $\begin{array}{l}\text { Diabetes } \\
\text { mellitus } \\
\text { type I or } \\
\text { type } 2\end{array}$ & Pharmaceutical & $\begin{array}{c}\text { Sweden }(2012 \\
\text { SEKs) }\end{array}$ & n.a/n.a. & I year & $\begin{array}{l}\text { Healthcare costs: } \\
\text { insulin costs, needles, } \\
\text { self-monitoring } \\
\text { blood glucose test } \\
\text { strips and lancets } \\
\text { costs, general } \\
\text { practitioner (GP) } \\
\text { visit, GP home visit, } \\
\text { and emergency } \\
\text { department visit } \\
\text { Social costs: } \\
\text { productivity losses }\end{array}$ & $\begin{array}{l}\text { Productivity } \\
\text { losses: human } \\
\text { capital approach } \\
\text { (paid time - } \\
\text { absenteeism) }\end{array}$ \\
\hline Saha $(20 \mid 3)^{54}$ & Diabetes & $\begin{array}{l}\text { Physical exercise } \\
\text { plus nutritional } \\
\text { recommendations }\end{array}$ & $\begin{array}{c}\text { Sweden } \\
(2012 \text { US\$) }\end{array}$ & $3 \% ; 3 \%$ & 85 years & $\begin{array}{l}\text { Health care costs: } \\
\text { medical treatment } \\
\text { costs, costs for } \\
\text { institutional health } \\
\text { care, } \\
\text { pharmaceuticals } \\
\text { Social costs: } \\
\text { productivity losses } \\
\text { and informal care } \\
\text { costs }\end{array}$ & $\begin{array}{l}\text { Productivity } \\
\text { losses: human } \\
\text { capital approach } \\
\text { (paid time due to } \\
\text { morbidity) } \\
\text { Informal care: } \\
\text { opportunity cost } \\
\text { method (non- } \\
\text { paid time) and } \\
\text { replacement cost } \\
\text { method }\end{array}$ \\
\hline $\begin{array}{l}\text { De Salas- } \\
\text { Cansado }(2012)^{33}\end{array}$ & Diabetes & Pharmaceutical & Spain $(2006 €)$ & n.a/n.a. & 12 weeks & $\begin{array}{l}\text { Healthcare costs: } \\
\text { drug and non-drug } \\
\text { treatments, medical } \\
\text { visits, hospitalizations } \\
\text { and diagnostic tests } \\
\text { Social costs: } \\
\text { productivity losses }\end{array}$ & $\begin{array}{l}\text { Productivity } \\
\text { losses: human } \\
\text { capital approach } \\
\text { (paid time - } \\
\text { absenteeism and } \\
\text { presenteeism) }\end{array}$ \\
\hline
\end{tabular}

(Continued) 
Table I (Continued).

\begin{tabular}{|c|c|c|c|c|c|c|c|}
\hline $\begin{array}{l}\text { First Author \& } \\
\text { Publication } \\
\text { Year }\end{array}$ & $\begin{array}{c}\text { Diabetes } \\
\text { Type }\end{array}$ & $\begin{array}{c}\text { Intervention } \\
\text { Type }\end{array}$ & $\begin{array}{l}\text { Country } \\
\text { and } \\
\text { Currency }\end{array}$ & $\begin{array}{c}\text { Discount } \\
\text { Rate } \\
\text { (Costs/ } \\
\text { Outcomes) }\end{array}$ & $\begin{array}{c}\text { Time } \\
\text { Horizon }\end{array}$ & Costs Included & $\begin{array}{l}\text { Method Used } \\
\text { for Calculating } \\
\text { Social Costs }\end{array}$ \\
\hline Kamble $(2012)^{69}$ & $\begin{array}{c}\text { Diabetes } \\
\text { mellitus } \\
\text { type I }\end{array}$ & Medical device & $\begin{array}{c}\text { United States } \\
\text { (2010US\$) }\end{array}$ & $3 \% ; 3 \%$ & 60 years & $\begin{array}{l}\text { Healthcare costs: } \\
\text { costs of glucose } \\
\text { meters and test } \\
\text { strips, lancets, } \\
\text { insulin, and provider } \\
\text { time to obtain annual } \\
\text { treatment costs, } \\
\text { costs of insulin } \\
\text { pumps, transmitters, } \\
\text { sensors, insertion } \\
\text { devices and other } \\
\text { pump suppliers } \\
\text { Social costs: } \\
\text { productivity losses }\end{array}$ & $\begin{array}{l}\text { Productivity } \\
\text { losses: human } \\
\text { capital approach } \\
\text { (paid time - } \\
\text { absenteeism) }\end{array}$ \\
\hline $\begin{array}{l}\text { Oostdam } \\
(2012)^{46}\end{array}$ & $\begin{array}{c}\text { Gestational } \\
\text { diabetes }\end{array}$ & $\begin{array}{c}\text { Non- } \\
\text { pharmaceutical } \\
\text { (exercise } \\
\text { intervention) }\end{array}$ & $\begin{array}{c}\text { Netherlands } \\
\text { (2009€) }\end{array}$ & n.a/n.a. & 32 weeks & $\begin{array}{l}\text { Healthcare costs: } \\
\text { visits to healthcare } \\
\text { providers, } \\
\text { medication } \\
\text { Social costs: } \\
\text { productivity losses } \\
\text { and informal care } \\
\text { costs }\end{array}$ & $\begin{array}{c}\text { Productivity } \\
\text { losses: human } \\
\text { capital approach } \\
\text { (paid time - } \\
\text { absenteeism) and } \\
\text { friction cost } \\
\text { method (paid } \\
\text { time - } \\
\text { absenteeism) } \\
\text { Informal care: n. } \\
\text { a. }\end{array}$ \\
\hline $\begin{array}{l}\text { Smith-Palmer } \\
(2012)^{56}\end{array}$ & $\begin{array}{l}\text { Diabetes } \\
\text { mellitus } \\
\text { type } 2\end{array}$ & Pharmaceutical & $\begin{array}{c}\text { Sweden } \\
\text { (20IOSEKs) }\end{array}$ & $3 \% ; 3 \%$ & $\begin{array}{l}\text { Lifetime } \\
\qquad(40 \\
\text { years })\end{array}$ & $\begin{array}{l}\text { Healthcare costs: } \\
\text { diabetes-related } \\
\text { complications costs, } \\
\text { medications, self- } \\
\text { monitoring blood } \\
\text { glucose tests costs, } \\
\text { treatment costs } \\
\text { Social costs: } \\
\text { productivity losses }\end{array}$ & $\begin{array}{l}\text { Productivity } \\
\text { losses: human } \\
\text { capital approach }\end{array}$ \\
\hline Greeley $(20 \mathrm{II})^{64}$ & $\begin{array}{l}\text { Diabetes } \\
\text { mellitus } \\
\text { type I }\end{array}$ & Screening & $\begin{array}{c}\text { United States } \\
\text { (2008US\$) }\end{array}$ & $3 \% ; 3 \%$ & $\begin{array}{l}10,20 \\
\text { and } 30 \\
\text { years }\end{array}$ & $\begin{array}{l}\text { Healthcare costs: } \\
\text { medications, test } \\
\text { strips, complications } \\
\text { costs } \\
\text { Social costs: informal } \\
\text { care costs }\end{array}$ & $\begin{array}{l}\text { Informal care: } \mathrm{n} \text {. } \\
\text { a. (paid time) }\end{array}$ \\
\hline
\end{tabular}

(Continued) 
Table I (Continued).

\begin{tabular}{|c|c|c|c|c|c|c|c|}
\hline $\begin{array}{l}\text { First Author \& } \\
\text { Publication } \\
\text { Year }\end{array}$ & $\begin{array}{l}\text { Diabetes } \\
\text { Type }\end{array}$ & $\begin{array}{c}\text { Intervention } \\
\text { Type }\end{array}$ & $\begin{array}{l}\text { Country } \\
\text { and } \\
\text { Currency }\end{array}$ & $\begin{array}{c}\text { Discount } \\
\text { Rate } \\
\text { (Costs/ } \\
\text { Outcomes) }\end{array}$ & $\begin{array}{c}\text { Time } \\
\text { Horizon }\end{array}$ & Costs Included & $\begin{array}{l}\text { Method Used } \\
\text { for Calculating } \\
\text { Social Costs }\end{array}$ \\
\hline Kasteng $(20 \mathrm{II})^{41}$ & $\begin{array}{l}\text { Diabetes } \\
\text { mellitus } \\
\text { type I or } \\
\text { type } 2\end{array}$ & Pharmaceutical & $\begin{array}{l}\text { Sweden } \\
\text { (2009SEKs) }\end{array}$ & $3 \% ; 3 \%$ & Lifetime & $\begin{array}{l}\text { Healthcare costs: } \\
\text { intervention drug } \\
\text { costs, complications } \\
\text { costs } \\
\text { Social costs: } \\
\text { productivity losses }\end{array}$ & $\begin{array}{l}\text { Productivity } \\
\text { losses: human } \\
\text { capital approach } \\
\text { (paid time - } \\
\text { absenteeism) }\end{array}$ \\
\hline Kuo $(201 \mathrm{I})^{70}$ & $\begin{array}{l}\text { Diabetes } \\
\text { mellitus } \\
\text { type I or } \\
\text { type } 2\end{array}$ & Care delivery & $\begin{array}{l}\text { United States } \\
(2010 \text { US } \$)\end{array}$ & $3 \% ; 3 \%$ & 20 years & $\begin{array}{l}\text { Healthcare costs: } \\
\text { endocrinologist, } \\
\text { registered/certified } \\
\text { nurse or diabetes } \\
\text { educator, exercise } \\
\text { physiologist, medical } \\
\text { assistant, rotated } \\
\text { staff, laboratory } \\
\text { tests, physician office } \\
\text { hours, complications } \\
\text { costs } \\
\text { Social costs: } \\
\text { productivity losses }\end{array}$ & $\begin{array}{c}\text { Productivity } \\
\text { losses: human } \\
\text { capital approach } \\
\text { (paid time) }\end{array}$ \\
\hline Patel $(20 \mathrm{II})^{47}$ & $\begin{array}{l}\text { Diabetes } \\
\text { mellitus } \\
\text { type I }\end{array}$ & $\begin{array}{c}\text { Health education } \\
\text { or behaviour }\end{array}$ & $\begin{array}{l}\text { United } \\
\text { Kingdom } \\
(2006 t)\end{array}$ & n.a/n.a. & I year & $\begin{array}{l}\text { Healthcare costs: } \\
\text { hospital inpatient and } \\
\text { outpatient services, } \\
\text { primary care } \\
\text { services, other } \\
\text { community-based } \\
\text { services, social } \\
\text { services, } \\
\text { medications, insulin- } \\
\text { related equipment, } \\
\text { other equipment and } \\
\text { adaptations and } \\
\text { intervention costs } \\
\text { Social costs: } \\
\text { productivity losses } \\
\text { and informal care } \\
\text { costs }\end{array}$ & $\begin{array}{c}\text { Productivity } \\
\text { losses: n.a. (paid } \\
\text { time - } \\
\text { absenteeism and } \\
\text { presenteeism - } \\
\text { and non-paid } \\
\text { time) } \\
\text { Informal care: } n \text {. } \\
\text { a. }\end{array}$ \\
\hline $\begin{array}{l}\text { Valentine } \\
(2011)^{60}\end{array}$ & $\begin{array}{c}\text { Diabetes } \\
\text { mellitus } \\
\text { type } 2\end{array}$ & Pharmaceutical & $\begin{array}{l}\text { Switzerland } \\
\text { (2008€) }\end{array}$ & $3 \% ; 3 \%$ & Lifetime & $\begin{array}{l}\text { Healthcare costs: } \\
\text { medications and } \\
\text { treatment costs, } \\
\text { complications costs } \\
\text { Social costs: } \\
\text { productivity losses }\end{array}$ & $\begin{array}{c}\text { Productivity } \\
\text { losses: human } \\
\text { capital approach } \\
\text { (paid time - } \\
\text { absenteeism) }\end{array}$ \\
\hline
\end{tabular}

(Continued) 
Table I (Continued).

\begin{tabular}{|c|c|c|c|c|c|c|c|}
\hline $\begin{array}{l}\text { First Author \& } \\
\text { Publication } \\
\text { Year }\end{array}$ & $\begin{array}{c}\text { Diabetes } \\
\text { Type }\end{array}$ & $\begin{array}{c}\text { Intervention } \\
\text { Type }\end{array}$ & $\begin{array}{l}\text { Country } \\
\text { and } \\
\text { Currency }\end{array}$ & $\begin{array}{c}\text { Discount } \\
\text { Rate } \\
\text { (Costs/ } \\
\text { Outcomes) }\end{array}$ & $\begin{array}{c}\text { Time } \\
\text { Horizon }\end{array}$ & Costs Included & $\begin{array}{l}\text { Method Used } \\
\text { for Calculating } \\
\text { Social Costs }\end{array}$ \\
\hline $\begin{array}{l}\text { Valentine } \\
(2011)^{59}\end{array}$ & $\begin{array}{l}\text { Diabetes } \\
\text { mellitus } \\
\text { type I }\end{array}$ & Pharmaceutical & $\begin{array}{l}\text { Sweden } \\
\text { (2006SEKs) }\end{array}$ & $3 \% ; 3 \%$ & 50 years & $\begin{array}{l}\text { Healthcare costs: } \\
\text { diabetes-related } \\
\text { complications costs, } \\
\text { pharmacy costs } \\
\text { Social costs: } \\
\text { productivity losses }\end{array}$ & $\begin{array}{l}\text { Productivity } \\
\text { losses: human } \\
\text { capital approach } \\
\text { (paid time - } \\
\text { absenteeism) }\end{array}$ \\
\hline Huang $(2010)^{68}$ & $\begin{array}{l}\text { Diabetes } \\
\text { mellitus } \\
\text { type I }\end{array}$ & $\begin{array}{l}\text { Diagnostic - } \\
\text { Medical device }\end{array}$ & $\begin{array}{l}\text { United States } \\
(2008 \text { US } \$)\end{array}$ & n.a/n.a. & Lifetime & $\begin{array}{l}\text { Healthcare costs: } \\
\text { intervention's } \\
\text { technology and } \\
\text { treatment costs, } \\
\text { standard glucose } \\
\text { monitoring costs, } \\
\text { routine office visits, } \\
\text { after-hours clinic } \\
\text { visits, emergency } \\
\text { room visits, } 9 \text { II calls, } \\
\text { and hospitalizations } \\
\text { Social costs: } \\
\text { productivity losses } \\
\text { and informal care } \\
\text { costs }\end{array}$ & $\begin{array}{c}\text { Productivity } \\
\text { losses: human } \\
\text { capital approach } \\
\text { (paid time - } \\
\text { absenteeism and } \\
\text { presenteeism) } \\
\text { Informal care: } \\
\text { opportunity cost } \\
\text { method (paid } \\
\text { time) }\end{array}$ \\
\hline Ismail $(2010)^{39}$ & $\begin{array}{l}\text { Diabetes } \\
\text { mellitus } \\
\text { type } 2\end{array}$ & $\begin{array}{c}\text { Non- } \\
\text { pharmacological } \\
\text { intervention }\end{array}$ & $\begin{array}{l}\text { United } \\
\text { Kingdom } \\
(2005 / 06 t)\end{array}$ & n.a./n.a. & I year & $\begin{array}{l}\text { Healthcare costs: } \\
\text { hospital inpatient and } \\
\text { outpatient services, } \\
\text { primary care } \\
\text { services, other } \\
\text { community-based } \\
\text { services, social } \\
\text { services, } \\
\text { medications, insulin- } \\
\text { related equipment, } \\
\text { other equipment and } \\
\text { adaptations and the } \\
\text { cost of the } \\
\text { interventions } \\
\text { Social costs: } \\
\text { productivity losses } \\
\text { and informal care } \\
\text { costs }\end{array}$ & $\begin{array}{l}\text { Productivity } \\
\text { losses: human } \\
\text { capital approach } \\
\text { (paid and non- } \\
\text { paid time) } \\
\text { Informal care: } \\
\text { opportunity cost } \\
\text { method (paid } \\
\text { and non-paid } \\
\text { time) }\end{array}$ \\
\hline
\end{tabular}

(Continued) 
Table I (Continued).

\begin{tabular}{|c|c|c|c|c|c|c|c|}
\hline $\begin{array}{l}\text { First Author \& } \\
\text { Publication } \\
\text { Year }\end{array}$ & $\begin{array}{c}\text { Diabetes } \\
\text { Type }\end{array}$ & $\begin{array}{c}\text { Intervention } \\
\text { Type }\end{array}$ & $\begin{array}{l}\text { Country } \\
\text { and } \\
\text { Currency }\end{array}$ & $\begin{array}{c}\text { Discount } \\
\text { Rate } \\
\text { (Costs/ } \\
\text { Outcomes) }\end{array}$ & $\begin{array}{c}\text { Time } \\
\text { Horizon }\end{array}$ & Costs Included & $\begin{array}{l}\text { Method Used } \\
\text { for Calculating } \\
\text { Social Costs }\end{array}$ \\
\hline $\begin{array}{l}\text { Gschwend } \\
(2009)^{37}\end{array}$ & $\begin{array}{l}\text { Diabetes } \\
\text { mellitus } \\
\text { type I }\end{array}$ & Pharmaceutical & $\begin{array}{l}\text { Belgium, } \\
\text { France, } \\
\text { Germany, Italy } \\
\text { and Spain } \\
(2006 €)\end{array}$ & $\begin{array}{l}\text { Belgium 3\% } \\
\text { costs, I.5\% } \\
\text { benefits; } \\
\text { France 3\% } \\
\text { both; } \\
\text { Germany 5\% } \\
\text { both; Italy 3\% } \\
\text { both; Spain } \\
6 \% \text { both }\end{array}$ & Lifetime & $\begin{array}{l}\text { Healthcare costs: } \\
\text { diabetes-related } \\
\text { complication costs, } \\
\text { medication (insulin) } \\
\text { and needles and } \\
\text { devices for self- } \\
\text { monitoring of blood } \\
\text { glucose } \\
\text { Social costs: } \\
\text { productivity losses }\end{array}$ & $\begin{array}{c}\text { Productivity } \\
\text { losses: human } \\
\text { capital approach }\end{array}$ \\
\hline Lindgren $(2007)^{45}$ & $\begin{array}{l}\text { Diabetes } \\
\text { mellitus } \\
\text { type } 2\end{array}$ & $\begin{array}{c}\text { Health education } \\
\text { or behaviour }\end{array}$ & $\begin{array}{c}\text { Sweden (2003 } \\
€)\end{array}$ & $3 \% ; 3 \%$ & n.a. & $\begin{array}{l}\text { Healthcare costs: } \\
\text { intervention costs, } \\
\text { physician visits, } \\
\text { nutritionist visits, } \\
\text { training sessions, } \\
\text { travel time, diabetes- } \\
\text { related complications } \\
\text { costs } \\
\text { Social costs: } \\
\text { productivity losses }\end{array}$ & $\begin{array}{l}\text { Productivity } \\
\text { losses: human } \\
\text { capital approach } \\
\text { (paid time - } \\
\text { absenteeism) }\end{array}$ \\
\hline $\begin{array}{l}\text { Valentine } \\
(2006)^{72}\end{array}$ & Diabetes & Pharmaceutical & $\begin{array}{c}\text { United States } \\
\text { (2002US\$) }\end{array}$ & $3 \% ; 3 \%$ & 35 years & $\begin{array}{l}\text { Healthcare costs: } \\
\text { treatment, diabetes- } \\
\text { related } \\
\text { complications, } \\
\text { medication costs } \\
\text { Social costs: } \\
\text { productivity losses }\end{array}$ & $\begin{array}{l}\text { Productivity } \\
\text { losses: human } \\
\text { capital approach } \\
\text { (paid time - } \\
\text { absenteeism) }\end{array}$ \\
\hline Eddy $(2005)^{63}$ & Diabetes & $\begin{array}{c}\text { Health education } \\
\text { or behaviour }\end{array}$ & $\begin{array}{c}\text { United States } \\
\text { (2000US\$) }\end{array}$ & $3 \% ; 3 \%$ & 30 years & $\begin{array}{l}\text { Healthcare costs: } \\
\text { hospital admissions } \\
\text { and emergency } \\
\text { department visits, } \\
\text { office and clinic visits, } \\
\text { tests and discrete } \\
\text { procedures, } \\
\text { medications and } \\
\text { ongoing programs } \\
\text { Social costs: } \\
\text { productivity losses }\end{array}$ & $\begin{array}{l}\text { Productivity } \\
\text { losses: human } \\
\text { capital approach } \\
\text { (paid time - } \\
\text { absenteeism and } \\
\text { presenteeism) }\end{array}$ \\
\hline
\end{tabular}

(Continued) 
Table I (Continued).

\begin{tabular}{|c|c|c|c|c|c|c|c|}
\hline $\begin{array}{l}\text { First Author \& } \\
\text { Publication } \\
\text { Year }\end{array}$ & $\begin{array}{c}\text { Diabetes } \\
\text { Type }\end{array}$ & $\begin{array}{c}\text { Intervention } \\
\text { Type }\end{array}$ & $\begin{array}{l}\text { Country } \\
\text { and } \\
\text { Currency }\end{array}$ & $\begin{array}{c}\text { Discount } \\
\text { Rate } \\
\text { (Costs/ } \\
\text { Outcomes) }\end{array}$ & $\begin{array}{c}\text { Time } \\
\text { Horizon }\end{array}$ & Costs Included & $\begin{array}{l}\text { Method Used } \\
\text { for Calculating } \\
\text { Social Costs }\end{array}$ \\
\hline Herman $(2005)^{67}$ & $\begin{array}{l}\text { Diabetes } \\
\text { mellitus } \\
\text { type } 2\end{array}$ & $\begin{array}{l}\text { Health education } \\
\text { intervention - } \\
\text { Pharmaceutical }\end{array}$ & $\begin{array}{c}\text { United States } \\
\text { (2000US\$) }\end{array}$ & $3 \% ; 3 \%$ & Lifetime & $\begin{array}{l}\text { Healthcare costs: } \\
\text { intervention costs, } \\
\text { diabetes-related } \\
\text { complication costs, } \\
\text { physician visits, } \\
\text { hospitalizations } \\
\text { Social costs: } \\
\text { productivity losses }\end{array}$ & $\begin{array}{l}\text { Productivity } \\
\text { losses: n.a. }\end{array}$ \\
\hline Rosen $(2005)^{71}$ & Diabetes & Pharmaceutical & $\begin{array}{c}\text { United States } \\
\text { (2003US\$) }\end{array}$ & $3 \% ; 3 \%$ & Lifetime & $\begin{array}{l}\text { Healthcare costs: } \\
\text { intervention costs, } \\
\text { diabetes-related } \\
\text { complication costs, } \\
\text { ongoing costs of } \\
\text { care, medication } \\
\text { costs } \\
\text { Social costs: } \\
\text { productivity losses } \\
\text { and informal care } \\
\text { costs }\end{array}$ & $\begin{array}{l}\text { Productivity } \\
\text { losses: } n . a . \\
\text { Informal care: } n \text {. } \\
\text { a. }\end{array}$ \\
\hline $\begin{array}{l}\text { The Diabetes } \\
\text { Prevention } \\
\text { Program } \\
\text { Research Group } \\
(2003)^{65}\end{array}$ & $\begin{array}{l}\text { Diabetes } \\
\text { mellitus } \\
\text { type } 2\end{array}$ & $\begin{array}{l}\text { Pharmaceutical - } \\
\text { Health education } \\
\text { or behaviour }\end{array}$ & $\begin{array}{c}\text { United States } \\
\text { (2000US\$) }\end{array}$ & $3 \% ; 3 \%$ & 3 years & $\begin{array}{l}\text { Healthcare costs: } \\
\text { intervention costs, } \\
\text { side effects of the } \\
\text { intervention, care } \\
\text { outside the } \\
\text { prevention program } \\
\text { (hospital, emergency } \\
\text { room, urgent care, } \\
\text { and outpatient } \\
\text { services; telephone } \\
\text { calls to health care } \\
\text { providers; and } \\
\text { prescription } \\
\text { medications), travel } \\
\text { costs } \\
\text { Social costs: } \\
\text { productivity losses }\end{array}$ & $\begin{array}{c}\text { Productivity } \\
\text { losses: human } \\
\text { capital approach } \\
\text { (paid time - } \\
\text { absenteeism) }\end{array}$ \\
\hline
\end{tabular}

(Continued) 
Table I (Continued).

\begin{tabular}{|c|c|c|c|c|c|c|c|}
\hline $\begin{array}{l}\text { First Author \& } \\
\text { Publication } \\
\text { Year }\end{array}$ & $\begin{array}{c}\text { Diabetes } \\
\text { Type }\end{array}$ & $\begin{array}{c}\text { Intervention } \\
\text { Type }\end{array}$ & $\begin{array}{l}\text { Country } \\
\text { and } \\
\text { Currency }\end{array}$ & $\begin{array}{l}\text { Discount } \\
\text { Rate } \\
\text { (Costs/ } \\
\text { Outcomes) }\end{array}$ & $\begin{array}{c}\text { Time } \\
\text { Horizon }\end{array}$ & Costs Included & $\begin{array}{l}\text { Method Used } \\
\text { for Calculating } \\
\text { Social Costs }\end{array}$ \\
\hline $\begin{array}{l}\text { Almbrand } \\
(2000)^{30}\end{array}$ & $\begin{array}{l}\text { Diabetes } \\
\text { mellitus } \\
\text { type I or } \\
\text { type } 2\end{array}$ & Pharmaceutical & $\begin{array}{c}\text { Sweden (1999 } \\
€)\end{array}$ & $3 \% ; 3 \%$ & Lifetime & $\begin{array}{l}\text { Healthcare costs: } \\
\text { medication costs, } \\
\text { hospitalizations, } \\
\text { post-hospital } \\
\text { discharge costs, } \\
\text { diagnostic and } \\
\text { monitoring } \\
\text { procedures and } \\
\text { tests, and outpatient } \\
\text { visits } \\
\text { Social costs: } \\
\text { productivity losses }\end{array}$ & $\begin{array}{l}\text { Productivity } \\
\text { losses: human } \\
\text { capital approach } \\
\text { (paid time) }\end{array}$ \\
\hline
\end{tabular}

2 and 26). In estimation number $2,{ }^{32}$ when social costs were considered, the assessed intervention was no longer cost-saving, as it was from the healthcare perspective. Informal care was included only in two studies ${ }^{55,62}$ and productivity losses in six of them. All the interventions were focused on the assessment of educational or behavioural programmes or medical devices, and the time horizons were quite similar. Most of the interventions in which the inclusion of social costs modified the conclusions were medical devices interventions, ${ }^{40,51,52,62}$ whereas another two were health education or behaviour programmes $^{32,63}$ and another was a surgical procedure. ${ }^{55}$

11 estimations from 9 studies provided changes in the ICUR results but not in the conclusions of the economic assessment. $^{31,34,42,45,59-61,66,76}$ In 9 of them (estimations numbers $10,15,28,29,39,51,91,92$ and 102), the new intervention was already cost-effective (the ICUR was below the threshold) from the healthcare perspective but it dominated the comparator when the social costs were considered. Two estimations from one study reported lower QALYs in the treatment group and also lower costs when the healthcare perspective was considered, with the new intervention ICUR lying above the acceptance threshold. ${ }^{34}$ When the societal approach was considered, the ICUR resulted in negative values, as the assessed intervention became dominated due to its positive incremental costs and lower health gains, against its comparator. Finally, in the results of the economic assessment, we observed one change that was the opposite of the rest when the societal perspective was considered. A novel pharmaceutical treatment dominated the comparator treatment when the healthcare perspective was considered, but when the societal perspective was used, the intervention did not dominate the comparator, but remained cost-effective according to the threshold stated by the authors (estimation number $47^{42}$ ).

Figures 2 and 3 show the dispersion of the costs and QALYs of the 110 economic evaluation estimations included, according to the perspective applied. Both figures show the economic evaluation results of the assessed interventions, reporting the incremental health gains measured in QALYs in the horizontal axis and the incremental costs in the vertical axis. The dots located in the upperright quadrant represent those interventions with higher costs than their comparator but with gains in health results (better HRQoL) as well. Moreover, two frequently used reference values of cost-effectiveness thresholds $(30,000$ and 50,000 euros per QALY) are also provided, which are represented by the two blue diagonals. If dots were above those thresholds mean the interventions that would not be implemented since, when being compared with their comparator, their ICUR is higher than the threshold considered as a reference of willing to pay by the healthcare provider/ society. Conversely, any dot below those lines denotes a healthcare intervention that would be indeed implemented. Although minor differences might be observed, there seems to be a higher number of dots below the $\mathrm{X}$ axis (lower incremental costs) in Figure 3, which represents the societal perspective, than in Figure 2, which corresponds to the healthcare perspective. 


\begin{tabular}{|c|c|c|c|c|c|c|c|}
\hline 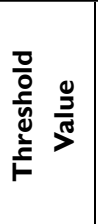 & & 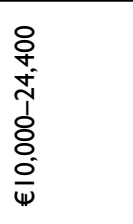 & 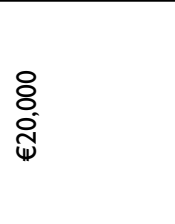 & $\begin{array}{l}8 \\
: \\
\stackrel{i}{w}\end{array}$ & $\begin{array}{l}\text { ¿ } \\
\text { ष্் }\end{array}$ & $\begin{array}{l}\text { ¿ } \\
\text { ष্் }\end{array}$ & 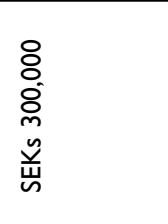 \\
\hline 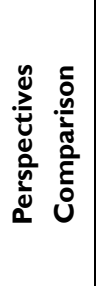 & 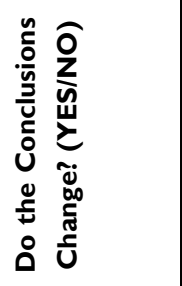 & 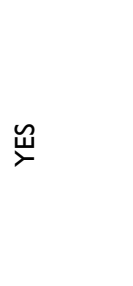 & 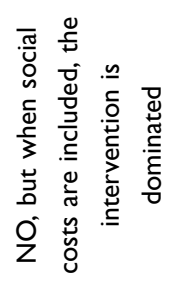 & 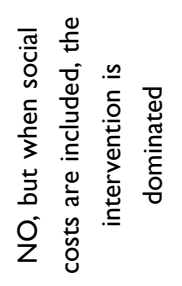 & 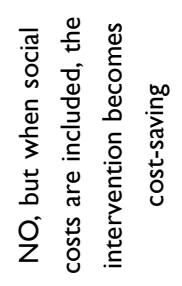 & 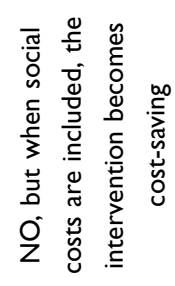 & 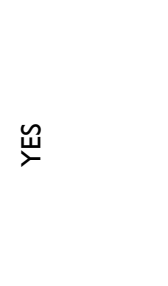 \\
\hline \multirow{4}{*}{ 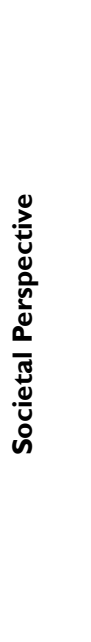 } & 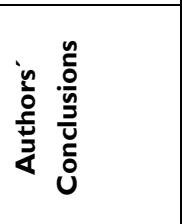 & 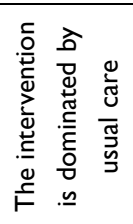 & 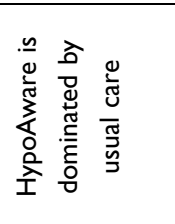 & 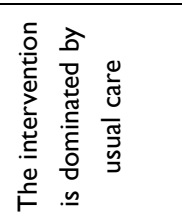 & 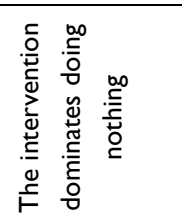 & 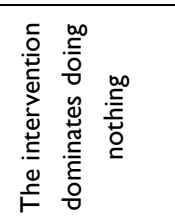 & 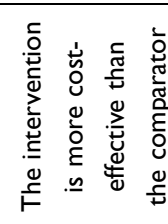 \\
\hline & 号 总 & $\frac{a}{a}$ & $\begin{array}{l}\stackrel{0}{ } \\
\infty \\
\infty \\
\infty \\
\infty\end{array}$ & $\underset{\substack{M \\
\infty}}{\stackrel{1}{\bar{p}}}$ & ষ্ণ & $\frac{8}{1}$ & $\begin{array}{l}\stackrel{+}{0} \\
\text { i } \\
\text { ָे }\end{array}$ \\
\hline & ¿ֶ̃ & $\begin{array}{l}\text { ọ } \\
\text { ọ }\end{array}$ & $\begin{array}{l}\infty \\
\stackrel{0}{0} \\
\stackrel{0}{0}\end{array}$ & ס̊̀ & চ̊̀口 & চ̊̀ & \\
\hline & Uू̆ & గొڤ & $\stackrel{\infty}{R}$ & $\stackrel{\infty}{\circledR}$ & o̊ & $\bar{o}$ & 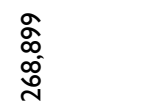 \\
\hline \multirow{4}{*}{ 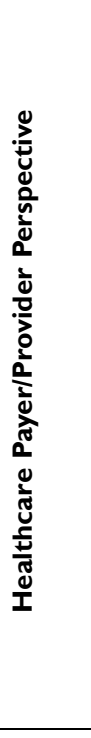 } & 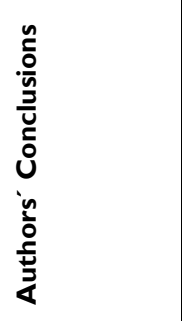 & 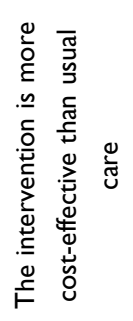 & 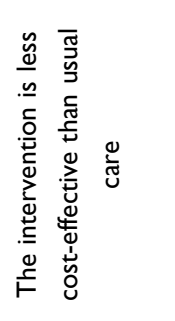 & 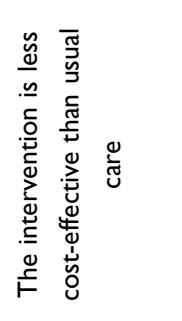 & 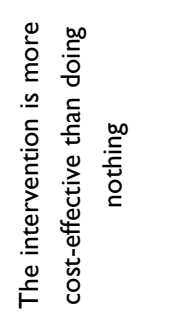 & 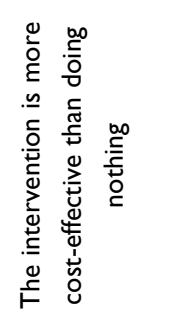 & 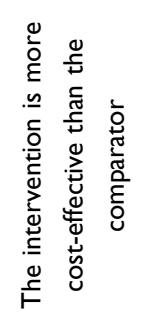 \\
\hline & 号 总 & & 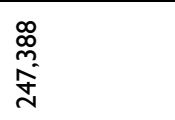 & $\frac{\stackrel{p}{m}}{m}$ & 0 & 0 & $\begin{array}{l}\stackrel{\circ}{+} \\
\stackrel{\infty}{\sim} \\
\tilde{m}\end{array}$ \\
\hline & $\stackrel{n}{\check{c}}$ & $\begin{array}{l}\text { ou } \\
\text { ọ }\end{array}$ & $\stackrel{\infty}{\circ}$ & ס̊. & চо̊ & 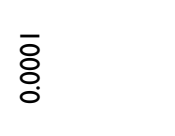 & o̊ \\
\hline & Ư⿱艹⿹勹巳 & $\ddot{\sim}$ & $\frac{\hat{\sigma}}{1}$ & $\frac{\hat{\sigma}}{1}$ & 0 & 0 & 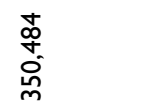 \\
\hline & 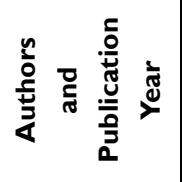 & 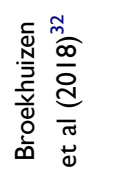 & 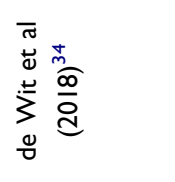 & 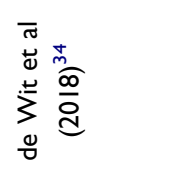 & 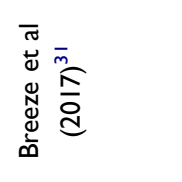 & 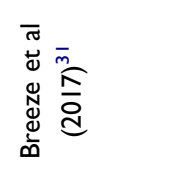 & 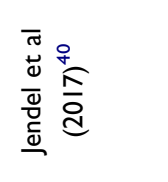 \\
\hline 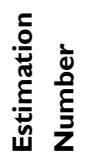 & & $N$ & $\sigma$ & in & 으 & $\underline{\underline{n}}$ & $\underline{\infty}$ \\
\hline
\end{tabular}




\begin{tabular}{|c|c|c|c|c|c|c|c|}
\hline 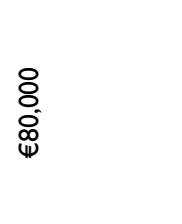 & 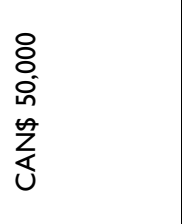 & 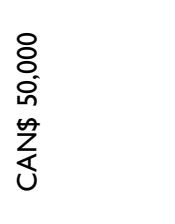 & 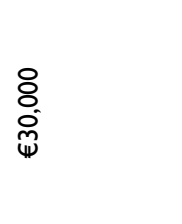 & $\begin{array}{l}8 \\
8 \\
8 \\
\frac{8}{1} \\
8 \\
0 \\
0 \\
\leftrightarrow\end{array}$ & 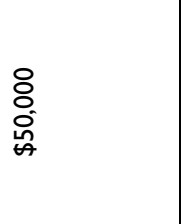 & $\begin{array}{l}8 \\
0 \\
0 \\
0 \\
\leftrightarrow\end{array}$ & 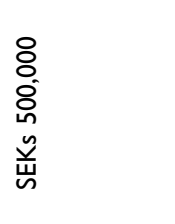 \\
\hline$\underset{\check{\nu}}{\breve{\nu}}$ & 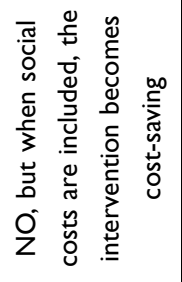 & 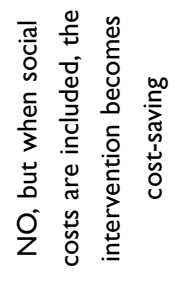 & $\underset{\check{\nu}}{\breve{u}}$ & 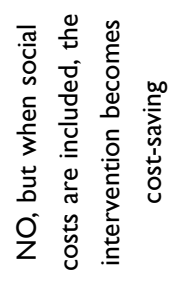 & $\underset{\check{\nu}}{\breve{\partial}}$ & $\underset{\breve{\nu}}{\breve{~}}$ & 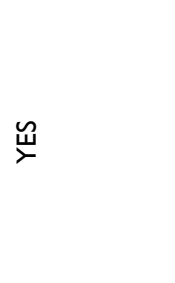 \\
\hline 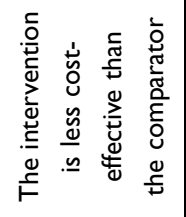 & 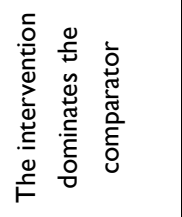 & 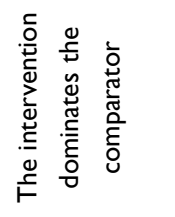 & 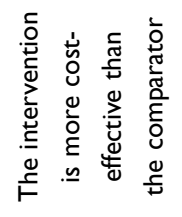 & 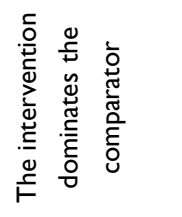 & 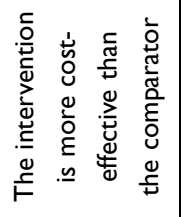 & 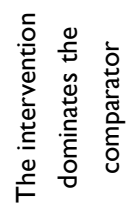 & 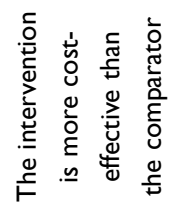 \\
\hline \begin{tabular}{l}
$\bar{\infty}$ \\
\multirow{+}{\alpha}{} \\
$\stackrel{\alpha}{\alpha}$
\end{tabular} & 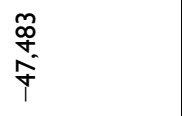 & $\begin{array}{l}\bar{m} \\
\underset{p}{\tilde{p}}\end{array}$ & $\begin{array}{l}\text { n్ } \\
\infty \\
\text { iे }\end{array}$ & $\begin{array}{l}\stackrel{0}{0} \\
\infty \\
\stackrel{0}{1} \\
1\end{array}$ & $\begin{array}{l}\infty \\
\stackrel{\infty}{\alpha} \\
\infty \\
\text { p }\end{array}$ & $\begin{array}{l}\frac{m}{0} \\
\frac{0}{0}\end{array}$ & $\begin{array}{l}\overline{\hat{n}} \\
\stackrel{n}{0} \\
\text { non }\end{array}$ \\
\hline તָ & $\stackrel{t}{0}$ & 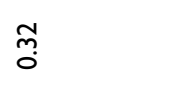 & $\stackrel{\widehat{\infty}}{=}$ & $\begin{array}{l}\bar{\alpha} \\
\stackrel{\alpha}{\circ} \\
\circ\end{array}$ & $\frac{\stackrel{\infty}{\infty}}{\stackrel{\infty}{0}}$ & $\frac{\stackrel{\infty}{\stackrel{\infty}{ড}}}{\stackrel{0}{0}}$ & $\stackrel{\circ}{i}$ \\
\hline 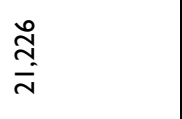 & $\begin{array}{l}\infty \\
\alpha \\
\frac{\infty}{1}\end{array}$ & $\begin{array}{l}\frac{\Delta}{E} \\
\overline{\bar{I}}\end{array}$ & 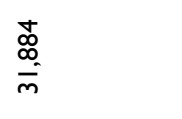 & $\begin{array}{l}\hat{\mathbf{o}} \\
\text { o } \\
\text { ○े } \\
1\end{array}$ & $\stackrel{\text { nㅡㅁ }}{\text { h }}$ & $\frac{\hat{\mathrm{o}}}{i}$ & $\begin{array}{l}\text { ऽ. } \\
\text { ๙े } \\
\text { సे }\end{array}$ \\
\hline 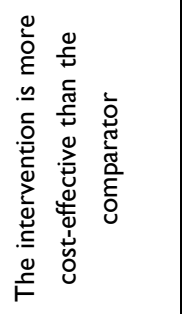 & 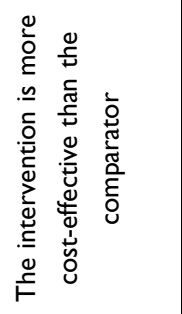 & 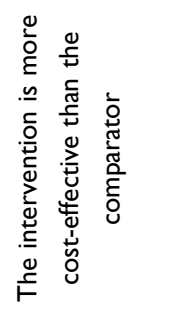 & 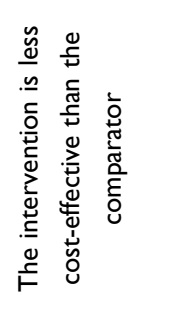 & 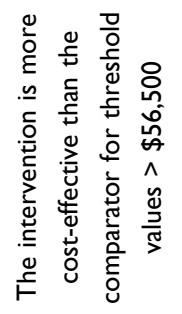 & 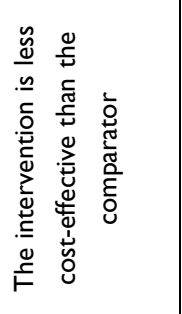 & 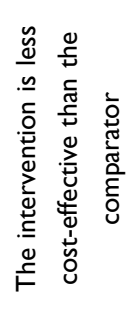 & 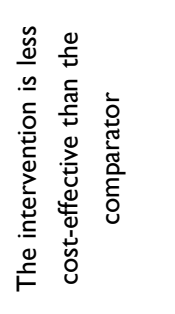 \\
\hline $\begin{array}{l}\frac{v}{m} \\
\hat{n}\end{array}$ & $\begin{array}{l}\dot{J} \\
\dot{\sigma} \\
\dot{d}\end{array}$ & $\begin{array}{l}\frac{\nabla}{\sigma} \\
\bar{d} \\
\dot{m}\end{array}$ & $\frac{\tilde{n}}{\dot{0}}$ & $\begin{array}{l}\text { 号 } \\
\text { ó } \\
\text { n̊ }\end{array}$ & 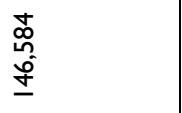 & $\frac{\infty}{\stackrel{\infty}{\text { Uీ }}}$ & $\begin{array}{l}\text { ڤ̊ } \\
\text { í } \\
\text { ஸे }\end{array}$ \\
\hline તุ & ț & $\underset{\tilde{m}}{0}$ & $\underline{\underline{\infty}}$ & $\begin{array}{l}\bar{\alpha} \\
\stackrel{\alpha}{\circ} \\
\circ\end{array}$ & $\frac{\stackrel{\infty}{\infty}}{\stackrel{\infty}{0}}$ & $\frac{\stackrel{\infty}{\stackrel{\infty}{\sim}}}{\stackrel{0}{0}}$ & $\stackrel{\circ}{i}$ \\
\hline 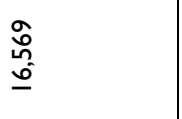 & ఖ్ & $\underset{\substack{\mathfrak{f} \\
=}}{ }$ & $\begin{array}{l}\bar{D} \\
\stackrel{\infty}{.} \\
\omega_{m}\end{array}$ & $\begin{array}{l}\stackrel{\circ}{0} \\
\text { ஸे } \\
\text { மீ }\end{array}$ & $\begin{array}{l}\stackrel{\infty}{\infty} \\
\infty \\
\infty \\
\infty\end{array}$ & $\begin{array}{l}\stackrel{\infty}{w} \\
\stackrel{m}{\underline{m}}\end{array}$ & $\frac{\frac{8}{\sigma}}{\frac{\sigma}{\sigma}}$ \\
\hline 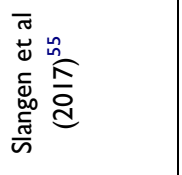 & 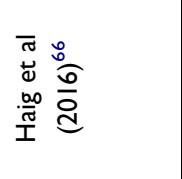 & 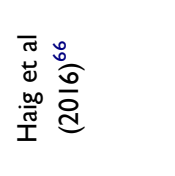 & 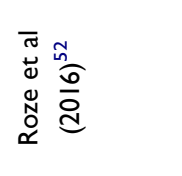 & 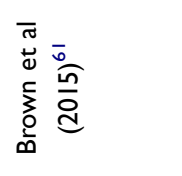 & 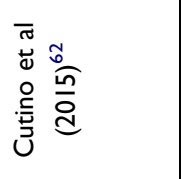 & 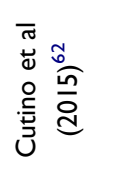 & 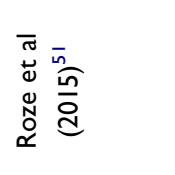 \\
\hline$\stackrel{\text { N }}{ }$ & $\stackrel{\sim}{\sim}$ & নి & $\hat{m}$ & 㝏 & 우 & $\bar{\sigma}$ & 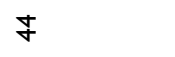 \\
\hline
\end{tabular}




\begin{tabular}{|c|c|c|c|c|c|c|c|}
\hline 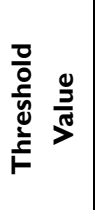 & & 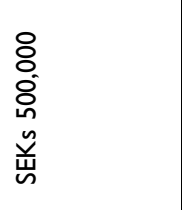 & $\begin{array}{l}8 \\
\stackrel{8}{0} \\
\text { Nn } \\
\leftrightarrow\end{array}$ & 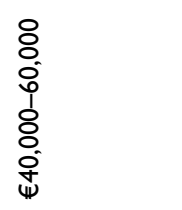 & 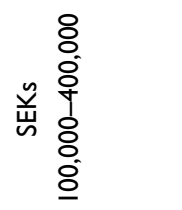 & 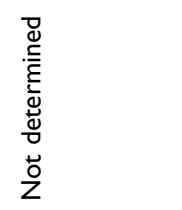 & $\begin{array}{l}8 \\
\frac{8}{4} \\
\frac{8}{4}\end{array}$ \\
\hline 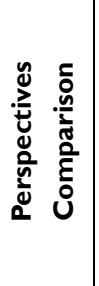 & 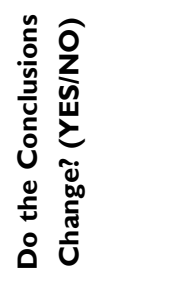 & 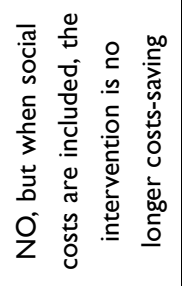 & 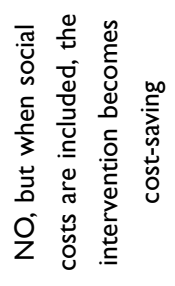 & 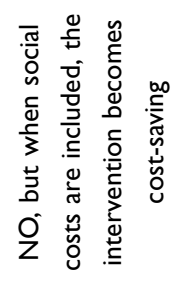 & 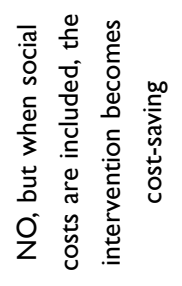 & 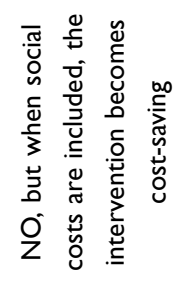 & $\underset{\breve{x}}{\breve{u}}$ \\
\hline \multirow{4}{*}{ 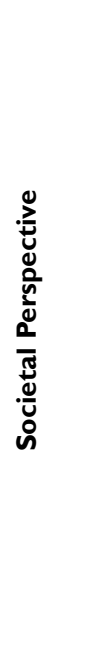 } & 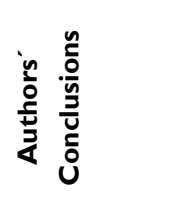 & 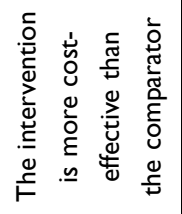 & 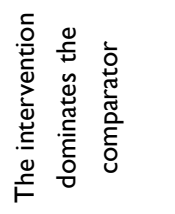 & 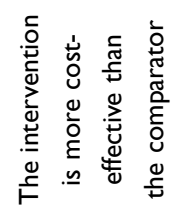 & 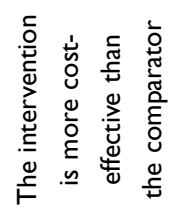 & 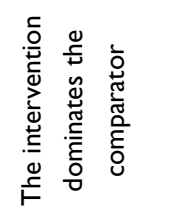 & 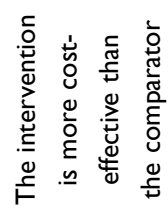 \\
\hline & 号 离 & 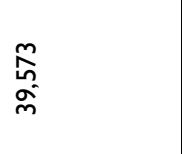 & $\frac{8}{0}$ & 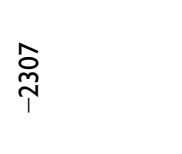 & $\frac{\frac{n}{n}}{\frac{n}{n}}$ & $\begin{array}{l}\stackrel{\sim}{i} \\
\stackrel{i}{i}\end{array}$ & \begin{tabular}{l}
$\infty$ \\
\multirow{0}{0}{} \\
i
\end{tabular} \\
\hline & $\stackrel{n}{\check{\delta}}$ & $\frac{n}{0}$ & $\bar{o}$ & $\frac{n}{0}$ & กิน & กี & $\frac{\text { 옹 }}{0}$ \\
\hline & 岁 & 心̊ & $\begin{array}{l}\frac{n}{\alpha} \\
\frac{\sigma}{1}\end{array}$ & $\underset{p}{\stackrel{0}{p}}$ & $\frac{m}{\bar{c}}$ & $\stackrel{\substack{n \\
\infty}}{1}$ & ڤু \\
\hline \multirow{5}{*}{ 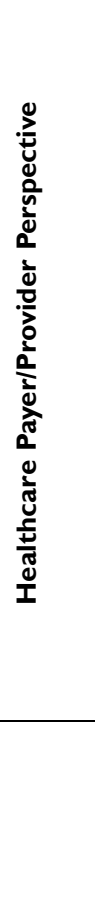 } & 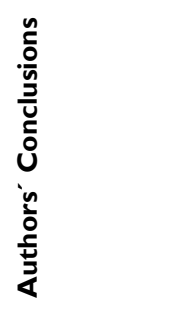 & 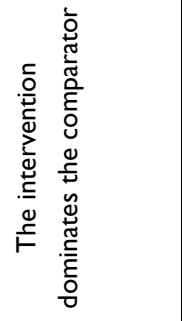 & 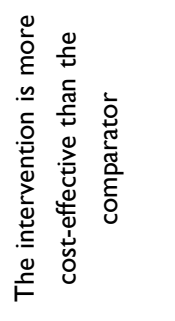 & 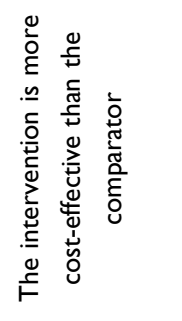 & 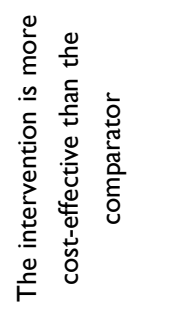 & 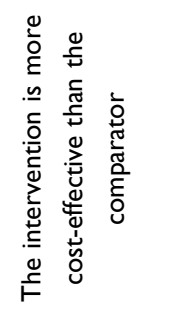 & 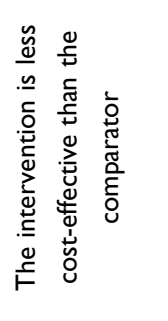 \\
\hline & 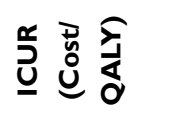 & $\underset{\substack{\hat{f} \\
\text { I }}}{1}$ & $\frac{n}{\stackrel{\circ}{*}}$ & 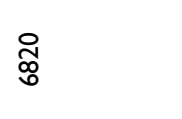 & $\begin{array}{l}\stackrel{\infty}{m} \\
\underset{m}{\sigma} \\
\sigma\end{array}$ & $\begin{array}{l}\stackrel{\sim}{\circ} \\
\stackrel{\infty}{\infty}\end{array}$ & $\begin{array}{l}\stackrel{8}{0} \\
\stackrel{m}{\underline{m}}\end{array}$ \\
\hline & $\stackrel{n}{\check{d}}$ & $\frac{n}{0}$ & $\overline{0}$ & $\frac{n}{0}$ & $\hat{n}$ & r̃ & $\frac{\text { 응 }}{\text { o }}$ \\
\hline & 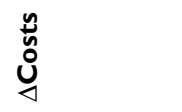 & $\frac{2}{\frac{1}{T}}$ & $\bar{\sim}$ & $\stackrel{\widetilde{\delta}}{\underline{\delta}}$ & $\frac{j}{i}$ & $\underset{\underline{\underline{o}}}{\underline{m}}$ & $\underset{\hat{N}}{\hat{i}}$ \\
\hline & 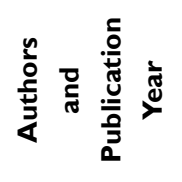 & 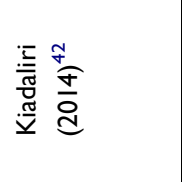 & 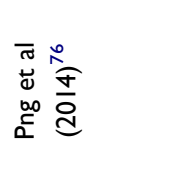 & 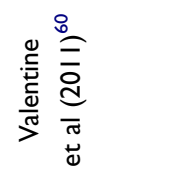 & 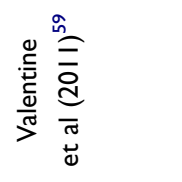 & 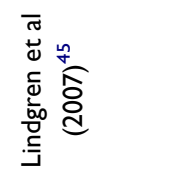 & 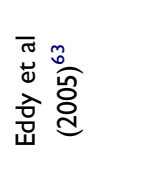 \\
\hline 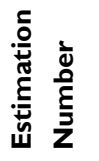 & & $\hat{f}$ & $\bar{n}$ & $\bar{\sigma}$ & $\sigma$ & $\underline{\underline{\delta}}$ & 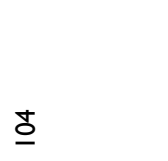 \\
\hline
\end{tabular}




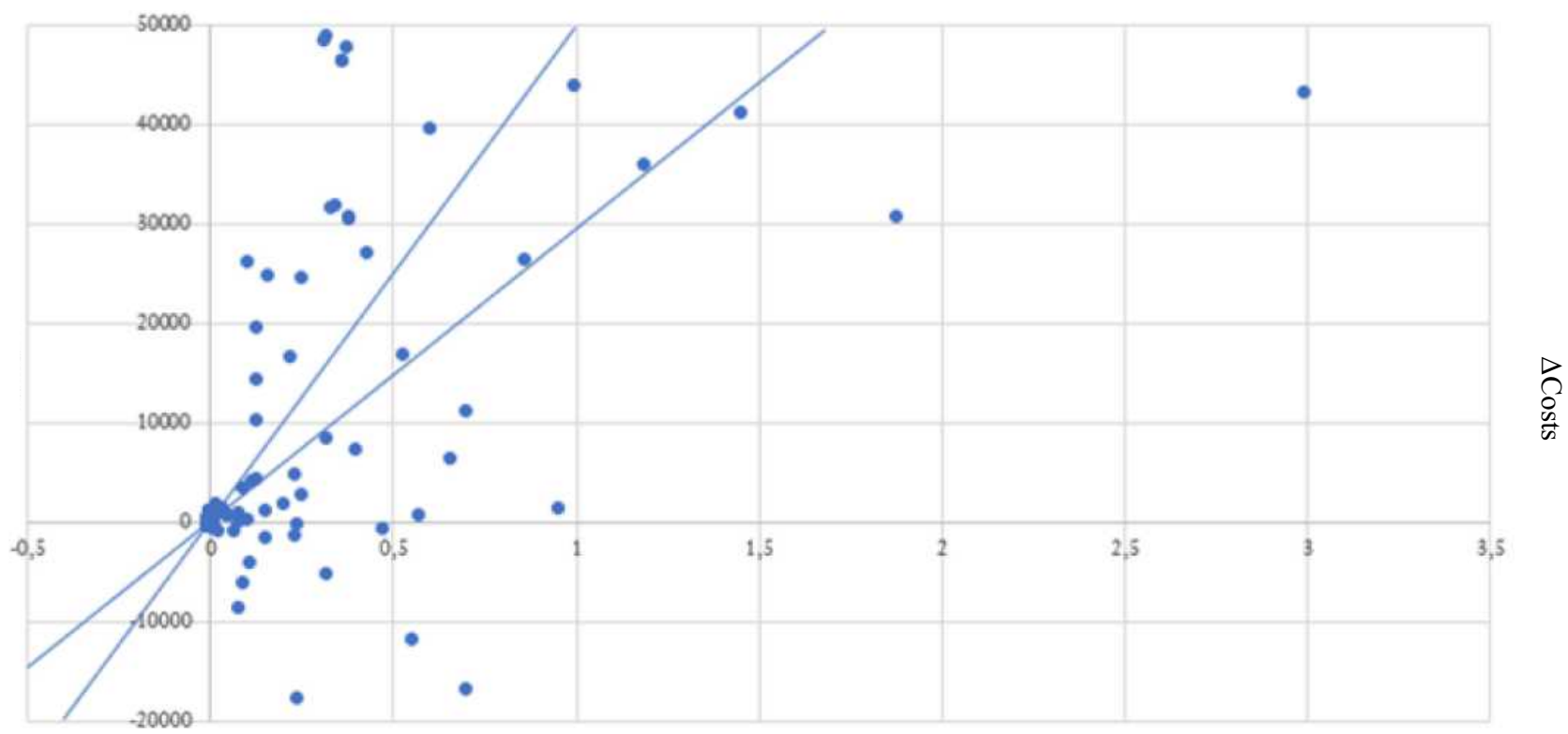

$\triangle \mathrm{QALYs}$

Figure 2 Incremental Cost-Utility Ratios from the healthcare perspective. For ease of comparison, results are shown in additional euros per additional QALY, applying the euro-currency exchange rates of the year of each record. The values were not updated to any base year since the efficiency thresholds applied as a usual reference are usually kept constant over several years. In this sense, and to facilitate the interpretation of the results of both panels, two vectors were drawn with the values of $€ 30,000 /$ $\mathrm{QALY}$ and $€ 50,000 / \mathrm{QALY}$ since they are frequently cited thresholds in the economic evaluation literature.

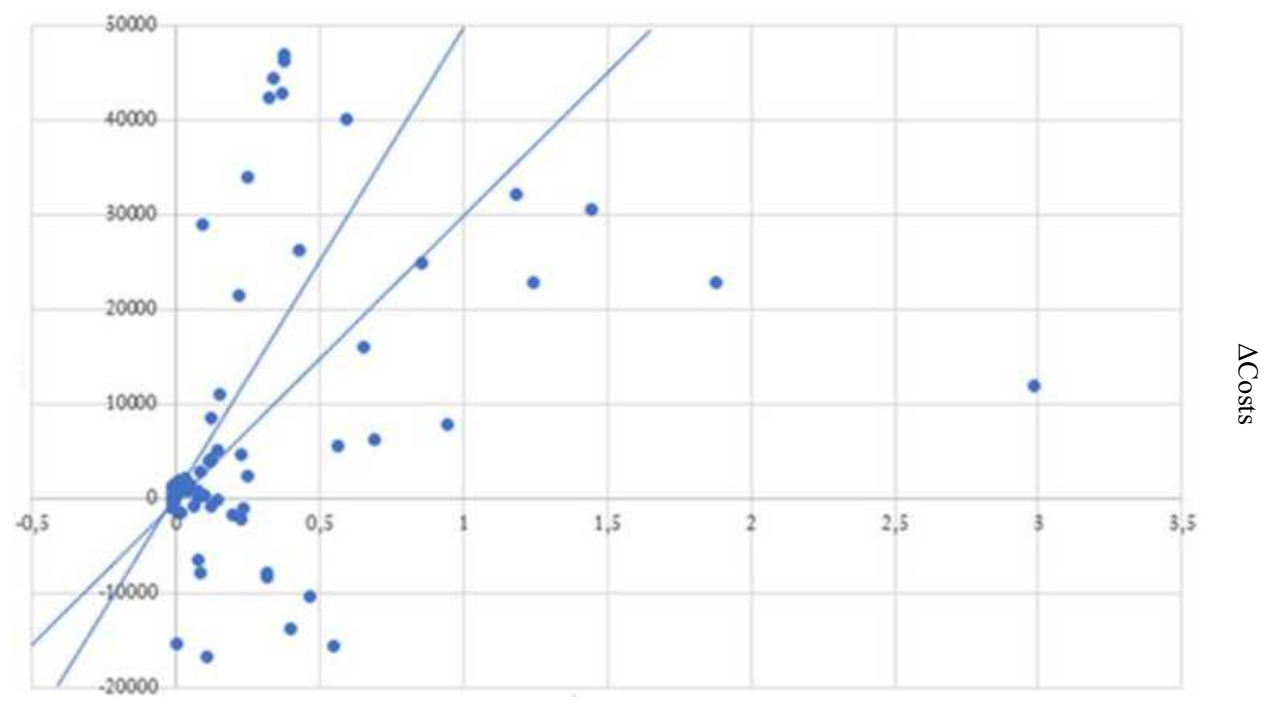

$\triangle$ QALYs

Figure 3 Incremental Cost-Utility Ratios from the societal perspective. For ease of comparison, results are shown in additional euros per additional QALY, applying the euro-currency exchange rates of the year of each record. The values were not updated to any base year since the efficiency thresholds applied as a usual reference are usually kept constant over several years. In this sense, and to facilitate the interpretation of the results of both panels, two vectors were drawn with the values of $€ 30,000 /$ QALY and $€ 50,000 / Q A L Y$ since they are frequently cited thresholds in the economic evaluation literature.

\section{Discussion}

Although many studies have systematically reviewed economic evaluations of diabetes-related interventions, our analysis builds on the existing literature by assessing the key role that social costs potentially play in the decisionmaking processes in diabetes-related healthcare technologies. Our findings showed that, of the 738 records that consisted of a full diabetes-associated economic 
evaluation, only 106 included social costs (14.36\%), from which 47 articles were finally selected in accordance with our inclusion criteria. An increasing number of national guidelines accept or even recommend the inclusion of a double perspective (societal perspective in addition to the healthcare payer/provider or the third-party payer perspective) as this obtains complementary results. ${ }^{77-83}$ However, of the forty-seven articles selected in our review, only sixteen applied both perspectives as the main viewpoints of the analyses. Moreover, our findings regarding the inclusion of social costs in all those articles that described an economic evaluation differed from those in similar work carried out on other diseases ${ }^{84}$ such as Alzheimer's disease, ${ }^{21}$ where the proportion of social costs in economic evaluations reached more than twothirds, and depression, where the figure was $42 \%{ }^{23}$ By contrast, for DM the percentage of economic evaluation studies which included social costs was close to that in the case of rare diseases, where it was only $11 \%{ }^{24}$

Moreover, from the 47 selected articles, 45 (96\%) included productivity losses whereas only 13 (28\%) included informal care costs. The scarce inclusion of informal care costs in diabetes-related economic evaluations and cost-of-illness studies has also been supported in the literature, which shows that only two articles from the 141 potential records in a recent review included informal care costs in the case of diabetes. Another recent review of economic evaluations carried out from 2013 to 2015 on several therapeutic areas, in which diabetes was not included, showed that the inclusion of informal care was limited (61 out of 484 potential records), but relevant, changing the conclusion of the economic evaluation or leading to cost savings. However, that review found that productivity losses and informal care costs were included in $86 \%$ and $23 \%$ respectively of the identified articles on musculoskeletal disorders or dysfunction, $94 \%$ and $47 \%$ respectively in the case of mental health problems, and $60 \%$ and $20 \%$ respectively in the case of infectious diseases. ${ }^{84}$ These figures point towards a lack of consensus in health technology assessment with respect to the costs which should be included, ${ }^{85}$ regardless of the therapeutic area analysed. On the other hand, the greater presence of productivity losses compared to informal care may be showing a bias in favour of reflecting costs that affect people of working age and against reflecting other costs that are related to older people. While it is true that the inclusion of labour losses has a greater tradition in the field of economic evaluation in general, and in the economic evaluation of healthcare interventions in particular, most people with diabetes are older people who are outside the labour market. Instead, according to most international studies, ${ }^{86-88}$ the non-professional or informal care which they receive is usually provided by their closest affective environment, more precisely, by their partner or their daughter. This means that a significant percentage of caregivers are not part of the labour force either. However, the opportunity cost incurred by caregivers represents a significant proportion of the social cost of diseases and should therefore be taken into account. On the other hand, current trends indicate that in the coming decades an important part of this informal care will be replaced or complemented by long-term professional care, ${ }^{89,90}$ which will have an important effect on public and household budgets.

The second purpose of the literature review was to assess whether the inclusion of social costs changed the conclusions regarding the adoption of the evaluated intervention. The conclusions were modified in eight out of the 110 estimations resulting from the 47 articles (7\%): six of them switched from being non-cost-effective from the healthcare payer/provider perspective to cost-effective when social costs were included. The results obtained were similar to those of other diseases such as Alzheimer's, in which the inclusion of social costs altered the conclusions in $11 \%$ of the economic evaluations included $^{21}$ but differed from the case of expensive drugs, where the change in the conclusions affected more than one third of the analyses. ${ }^{20}$ Some characteristics are shared among the studies whose conclusions changed after the inclusion of social costs and hence could be key drivers in the modification of conclusions: i) only productivity losses were entered as social costs, ${ }^{40,51,52,63}$ using the human capital approach for their valuation and with absenteeism being included in all of them; ${ }^{63}$ ii) the authors applied longer time horizons (from fifteen years to lifetime); iii) they were interventions targeting individuals with type 1 diabetes; and iv) the type of intervention, which in most cases was a medical device. ${ }^{40,51,52}$

Furthermore, without leading to a change in conclusions but providing them with additional support, nine estimations became dominant when social costs were included (estimation numbers 10, 15, 28, 29, 39, 51, 91, 92 and 102). The savings were almost negligible in two of these estimations (numbers 10 and 15), but in the other seven, cost savings were observed when the societal 
perspective was used. Moreover, these seven studies shared some common patterns: i) all of them referred to a pharmaceutical intervention among individuals with DM type 2; ii) longer time horizons, most of which were lifetime; and iii) productivity losses due to absenteeism, using the human capital approach for their valuation. A recent review of economic evaluations of different types of insulin, one of the main drugs used by people with diabetes, showed that its cost-effectiveness among individuals with type 2 diabetes was inconclusive. ${ }^{91-93}$ However, only in those studies where the authors performed the economic evaluation from a societal perspective (three from a total of the forty-three studies included), were the interventions dominant, ${ }^{36,94,95}$ highlighting the significant and favourable economic impact of including social costs in these interventions.

We also detected changes in the final conclusion but in the opposite way. This was the case of the results obtained in estimations 4 and 5 , in which de Wit et $\mathrm{al}^{34}$ assessed a health education or behaviour programme for people with DM type 1 or 2 during a six-month period. The results showed that when both productivity losses and informal care costs were included, the intervention was no longer cost-saving, whereas from the healthcare perspective, it was. These results, in addition to the two aforementioned studies that changed the conclusions from cost-effective to non-cost-effective ${ }^{55}$ or dominated, ${ }^{32}$ point towards a significant long-term effect on incremental costs from the introduction of social costs in the context of diabetes-related interventions.

Some limitations should be mentioned, especially in connection with methodological questions, which limit the comparability of studies. First, we may have missed some references due to our search strategy and the databases where it was launched. In this connection, we tried to avoid bias by using more than one dataset, and we aimed to retrieve economic evaluations using a cost-utility design by searching on both Medline and the CEA registry of Tufts University. The joint use of both literature databases can be explained by the following reasons: Medline contains more than 25 million references to journal articles about life sciences with a concentration on biomedicine, as well as the health-technology assessments (HTA) published in scientific journals. Although other literature databases could have been used, other previous works have shown that very few additional economic evaluations are found in databases other than Medline, while a number of papers may in fact be lost in other databases if substituted for Medline. ${ }^{96-99}$ In order to avoid loss of sensitivity in our search strategy, we also used the Cost-Effectiveness Analysis (CEA) Registry of Tufts University, which uses an algorithm also launched in Medline plus a systematic review process in which only cost-utility analyses are included. ${ }^{26,100}$ By using both sources, Medline and the CEA Registry, we were able to retrieve scientific papers that fulfilled our inclusion criteria. Secondly, it should be noted that we do not intend to perform again or re-interpret the economic evaluations included in the review, but, instead, we took into account the information provided by the original authors (ie the acceptability proposed after threshold) and their interpretations of the results. However, as Table A1 shows, such cost-effectiveness threshold values and conclusions might not be implicit and might be subject to debate. Thirdly, the comparability of studies related to the valuation of social costs might be compromised since no homogeneous methodology was observed among the studies included. For instance, with respect to the thirteen studies that included informal care costs, only six of them explicitly stated the method used to value informal care, the opportunity cost method being used in all of them, and both the opportunity cost and the replacement cost methods being used in one study. ${ }^{54}$ Moreover, only three of the studies provided the unit cost applied, and instead of this, the information provided by the others ranged from hourly wages ${ }^{39,55}$ to monthly costs, adjusted by age. ${ }^{62}$ Regarding productivity losses, from the forty-five included studies, four did not specify the method applied, and when mentioning the technique used, thirtytwo reported that they had applied the human capital approach to value productivity losses, four had used the friction cost method ${ }^{31,34,55,58}$ and one of them had used both. ${ }^{46}$ Of those using the friction cost approach, only one study provided detailed information about the length of the friction period (84 days) and the attribution of genderspecific incomes. ${ }^{34}$ From the productivity loss components, time paid for absenteeism was valued in twentysix works, and absenteeism and presenteeism in three articles. $^{33,63,68}$ Unpaid time was additionally included with paid time in three studies. ${ }^{34,39,47}$

We conclude by pointing out that despite the burden that social costs impose on diabetes, there is still a lack of evidence about the consequences of excluding them from economic evaluations of interventions targeting people with diabetes. The literature review performed shed new light on cost-utility analyses involving the assessment of diabetes-related interventions that include social costs, 
showing that consideration of informal care costs and/or productivity losses changed the results or conclusions in $18 \%$ of economic evaluations of diabetes (in 20 out of 110). Moreover, the inclusion of productivity losses in most of the selected articles (96\%) and of informal care costs in almost one third of them signals the burden that diabetes imposes on its sufferers, limiting their ability to work $^{11,13-15}$ or requiring a greater need for care. ${ }^{13,101,102}$ However, comparisons should be made with caution, as the different types of diabetes affect completely different profiles, with respect to age, state of health, healthcare use and lifestyle. ${ }^{103}$ While type 1 DM (5-10\% of all diabetes) usually starts in childhood or early adulthood and usually cannot be prevented, type 2 DM (90-95\% of all diabetes) is commonly developed during adulthood and old age and can be prevented or delayed by healthy lifestyles. ${ }^{104,105}$ Those different patient profiles should promote a change in the type of social costs included and their effect on the adoption of new healthcare technologies, as this literature review has shown. In view of the increasing worldwide prevalence of diabetes that is expected in the coming years, mainly of type 2 diabetes in older populations, ${ }^{106}$ and the fact that policymakers may need to balance the impact of new healthcare interventions on health budgets, incident cases of diabetes and equity of healthcare provision, the findings obtained here might be of help in directing the appropriate interventions to the right diabetes populations within their scope. In this sense, considering the age group mainly affected by type $2 \mathrm{DM}$ and its associated complications, which might impair their functional status and might result in a greater need for care, further research should take into account the inclusion of additional informal care costs in the analysis. This would help to bring new evidence closer to real clinical practice and would therefore help to provide policymakers with relevant and accurate information that would consider the effect of ageing on social services and non-formal resources aimed at fighting dependence in the performance of activities of daily living.

Moreover, since comparisons of current cost-utility analyses are challenging because of the various alternatives and methodologies used, further improvement is required, particularly in relation to explanations of the perspectives applied in the analysis, to the methodology applied to perform the economic evaluation and to the sources of utility and costs (especially in those studies where a lifetime is used, and a mathematical model is applied to simulate the progress of the illness). The inclusion of all types of costs and health effects from a societal perspective could serve as more detailed information to ease decision-making by policymakers, leading to a more efficient and equitable design of diabetes-related programmes and interventions which could eventually be implemented.

\section{Data Sharing Statement}

All data generated or analysed during this study are included in this published article (and its Appendix).

\section{Acknowledgments}

We would like to thank Luz María Peña Longobardo for the help provided throughout the review process and the manuscript writing, and Leocadio Rodríguez Mañas for his medical writing support.

The abstract of this paper was presented at the Virtual ISPOR Europe 2020 conference as a poster with interim findings. The poster's abstract was published in the journal Value in Health 2020; 23(2): S511-S512: https://doi.org/ 10.1016/j.jval.2020.08.632.

\section{Author Contributions}

All authors made a significant contribution to the work reported, whether that is in the conception, study design, execution, acquisition of data, analysis and interpretation, or in all these areas; took part in drafting, revising or critically reviewing the article; gave final approval of the version to be published; have agreed on the journal to which the article has been submitted; and agree to be accountable for all aspects of the work.

\section{Funding}

This project has received funding from the European Union's Horizon 2020 research and innovation programme, under grant agreement no. 779312.

\section{Disclosure}

BRS, IAR, JOM and JLB report to have received funding grants from the European Commission, during the conduct of the study. No other disclosures were reported by any of the authors.

\section{References}

1. Cho NH, Shaw JE, Karuranga S, et al. IDF diabetes atlas: global estimates of diabetes prevalence for 2017 and projections for 2045 . Diabetes Res Clin Pract. 2018;138:271-281. doi:10.1016/j. diabres.2018.02.023 
2. Collaborators GDaH. Global, regional, and national disabilityadjusted life-years (DALYs) for 315 diseases and injuries and healthy life expectancy (HALE), 1990-2015: a systematic analysis for the Global Burden of Disease Study 2015. Lancet Diabetes Endocrinol. 2016;388:1603-11658.

3. Association AD. Economic costs of diabetes in the U.S. in 2017. Diabetes Care. 2018;41(5):917-928. doi:10.2337/dci18-0007

4. Bommer C, Sagalova V, Heesemann E, et al. Global economic burden of diabetes in adults: projections from 2015 to 2030. Diabetes Care. 2018;41(5):963-970. doi:10.2337/dc17-1962

5. Li R, Bilik D, Brown MB, et al. Medical costs associated with type 2 diabetes complications and comorbidities. Am J Manag Care. 2013;19(5):421-430.

6. Zhuo X, Zhang P, Barker L, Albright A, Thompson TJ, Gregg E. The lifetime cost of diabetes and its implications for diabetes prevention. Diabetes Care. 2014;37(9):2557-2564. doi:10.2337/dc13-2484

7. Clarke PM, Glasziou P, Patel A, et al. Event rates, hospital utilization, and costs associated with major complications of diabetes: a multicountry comparative analysis. PLoS Med. 2010;7(2):e1000236. doi:10.1371/journal.pmed.1000236

8. de Lagasnerie G, Aguade AS, Denis P, Fagot-Campagna A, Gastaldi-Menager C. The economic burden of diabetes to French national health insurance: a new cost-of-illness method based on a combined medicalized and incremental approach. Eur J Health Econ. 2018;19(2):189-201. doi:10.1007/s10198-0170873-y

9. Munshi MN, Meneilli GS, Rodríguez-Mañas L, et al. Diabetes in ageing: pathways for developing the evidence base for clinical guidance. Lancet Diabetes Endocrinol. 2020;8(10):855-867.

10. Anderson JM. Achievable cost saving and cost-effective thresholds for diabetes prevention lifestyle interventions in people aged 65 years and older: a single-payer perspective. J Acad Nutr Diet. 2012;112(11):1747-1754. doi:10.1016/j.jand.2012.08.033

11. Tunceli O, Wade R, Gu T, Bouchard JR, Aagren M, Luo W. Cost of diabetes: comparison of disease-attributable and matched cohort cost estimation methods. Curr Med Res Opin. 2010;26 (8):1827-1834. doi:10.1185/03007995.2010.488544

12. Vicente-Herrero MT, Terradillos Garcia MJ, Capdevila Garcia LM, Ramirez Iniguez de la Torre MV, Lopez-Gonzalez AA. [Costs of temporary disability in Spain related to diabetes mellitus and its complications]. Endocrinol Nutr. 2013;60 (8):447-455. Spanish. doi:10.1016/j.endonu.2013.02.004

13. Hex N, Bartlett C, Wright D, Taylor M, Varley D. Estimating the current and future costs of type 1 and type 2 diabetes in the UK, including direct health costs and indirect societal and productivity costs. Diabet Med. 2012;29(7):855-862. doi:10.1111/j.14645491.2012.03698.x

14. Herquelot E, Guéguen A, Bonenfant S, Dray-Spira R. Impact of diabetes on work cessation: data from the GAZEL cohort study. Diabetes Care. 2011;34(6):1344-1349. doi:10.2337/dc10-2225

15. Rumball-Smith J, Barthold D, Nandi A, Heymann J. Diabetes associated with early labour-force exit: a comparison of sixteen high-income countries. Health Aff (Project Hope). 2014;3391:110-115. doi:10.1377/hlthaff.2013.0518

16. Bolin K, Gip C, Mork AC, Lindgren B. Diabetes, healthcare cost and loss of productivity in Sweden 1987 and 2005-a registerbased approach. Diabet Med. 2009;26(9):928-934. doi:10.1111/ j.1464-5491.2009.02786.x

17. Lopez-Bastida J, Boronat M, Moreno JO, Schurer W. Costs, outcomes and challenges for diabetes care in Spain. Global Health. 2013;9(1):17. doi:10.1186/1744-8603-9-17

18. Lopez-Bastida J, Lopez-Siguero JP, Oliva-Moreno J, et al. Social economic costs of type 1 diabetes mellitus in pediatric patients in Spain: CHRYSTAL observational study. Diabetes Res Clin Pract. 2017;127:59-69. doi:10.1016/j.diabres.2017.02.033
19. Goodrich K, Kaambwa B, Al-Janabi H. The inclusion of informal care in applied economic evaluation: a review. Value Health. 2012;15(6):975-981. doi:10.1016/j.jval.2012.05.009

20. Krol M, Papenburg J, Tan SS, Brouwer W, Hakkaart L. A noticeable difference? Productivity costs related to paid and unpaid work in economic evaluations on expensive drugs. Eur J Health Econ. 2016;17(4):391-402. doi:10.1007/s10198-015-0685-x

21. Peña-Longobardo LM, Rodríguez-Sánchez B, Oliva-Moreno J, Aranda-Reneo I, López-Bastida J. How relevant are social costs in economic evaluations? The case of Alzheimer's disease. Eur J Health Econ. 2019;20(8):1207-1236. doi:10.1007/s10198-01901087-6

22. Krol M, Papenburg J, van Exel J. Does including informal care in economic evaluations matter? A systematic review of inclusion and impact of informal care in cost-effectiveness studies. Pharmacoeconomics. 2015;33(2):123-135. doi:10.1007/s40273014-0218-y

23. Duevel JA, Hasemann L, Peña-Longobardo LM, et al. Considering the societal perspective in economic evaluations: a systematic review in the case of depression. Health Econ Rev. 2020;10(1). doi:10.1186/s13561-020-00288-7

24. Aranda-Reneo I, Rodriguez-Sanchez B, Peña-Longobardo LM, Oliva-Moreno J, Lopez-Bastida J. Can the consideration of social costs change the recommendation of economic evaluations in the field of rare diseases? An empirical analysis. Value Health. 2020. doi:10.1016/j.jval.2020.10.014

25. Methley AM, Campbell S, Chew-Graham C, McNally R, Cheraghi-Sohi S. PICO, PICOS and SPIDER: a comparison study of specificity and sensitivity in three search tools for qualitative systematic reviews. BMC Health Serv Res. 2014;14. doi:10.1186/s12913-014-0579-0

26. Thorat T, Cangelosi M, Neumann PJ. Skills of the trade: the tufts cost-effectiveness analysis registry. J Benefit-Cost Anal. 2012;3 (1):1-9. doi:10.1515/2152-2812.1114

27. Neumann PJ, Thorat T, Shi J, Saret CJ, Cohen JT. The changing face of the cost-utility literature, 1990-2012. Value Health. 2015;18(2):271-277. doi:10.1016/j.jval.2014.12.002

28. Moher D, Liberati A, Tetzlaff J, Altman DG; Group TP. Preferred reporting items for systematic reviews and meta-analyses: the PRISMA Statement. PLoS Med. 2009;6(7):e1000097. doi:10.1371/journal.pmed.1000097

29. OECD EaWHO. A System of Health Accounts 2011. Revised ed. Paris: OECD Publishing; 2017.

30. Almbrand B, Johannesson M, Sjostrand B, Malmberg K, Ryden L. Cost-effectiveness of intense insulin treatment after acute myocardial infarction in patients with diabetes mellitus; results from the DIGAMI study. Eur Heart J. 2000;21 (9):733-739. doi:10.1053/euhj.1999.1859

31. Breeze PR, Thomas C, Squires H, et al. Cost-effectiveness of population-based, community, workplace and individual policies for diabetes prevention in the UK. Diabet Med. 2017;34 (8):1136-1144. doi:10.1111/dme.13349

32. Broekhuizen K, Simmons D, Devlieger R, et al. Costeffectiveness of healthy eating and/or physical activity promotion in pregnant women at increased risk of gestational diabetes mellitus: economic evaluation alongside the DALI study, a European multicenter randomized controlled trial. Int J Behav Nutr Phys Act. 2018;15(1):23. doi:10.1186/s12966018-0643-y

33. de Salas-cansado M, Perez C, Saldana MT, et al. An economic evaluation of pregabalin versus usual care in the management of community-treated patients with refractory painful diabetic peripheral neuropathy in primary care settings. Prim Care Diabetes. 2012;6(4):303-312. doi:10.1016/j. pcd.2012.03.001 
34. de Wit M, Rondags S, van Tulder MW, Snoek FJ, Bosmans JE. Costeffectiveness of the psycho-educational blended (group and online) intervention HypoAware compared with usual care for people with Type 1 and insulin-treated Type 2 diabetes with problematic hypoglycaemia: analyses of a cluster-randomized controlled trial. Diabet Med. 2018;35(2):214-222. doi:10.1111/dme.13548

35. Ericsson A, Glah D, Lorenzi M, Jansen JP, Fridhammar A. Costeffectiveness of liraglutide versus lixisenatide as add-on therapies to basal insulin in type 2 diabetes. PLoS One. 2018;13(2): e0191953. doi:10.1371/journal.pone.0191953

36. Ericsson A, Pollock RF, Hunt B, Valentine WJ. Evaluation of the cost-utility of insulin degludec vs insulin glargine in Sweden. $J$ Med Econ. 2013;16(12):1442-1452. doi:10.3111/13696998 .2013 .852099

37. Gschwend MH, Aagren M, Valentine WJ. Cost-effectiveness of insulin detemir compared with neutral protamine Hagedorn insulin in patients with type 1 diabetes using a basal-bolus regimen in five European countries. J Med Econ. 2009;12(2):114-123. doi:10.3111/13696990903080344

38. Huetson P, Palmer JL, Levorsen A, Fournier M, Germe M, McLeod E. Cost-effectiveness of once daily GLP-1 receptor agonist lixisenatide compared to bolus insulin both in combination with basal insulin for the treatment of patients with type 2 diabetes in Norway. $J$ Med Econ. 2015;18(8):573-585. doi:10.3111/13696998.2015.1038271

39. Ismail K, Maissi E, Thomas S, et al. A randomised controlled trial of cognitive behaviour therapy and motivational interviewing for people with Type 1 diabetes mellitus with persistent sub-optimal glycaemic control: a Diabetes and Psychological Therapies (ADaPT) study. Health Technol Assess. 2010;14(22):1-101, iiiiv. doi:10.3310/hta14220

40. Jendle J, Smith-Palmer J, Delbaere A, et al. Cost-effectiveness analysis of sensor-augmented insulin pump therapy with automated insulin suspension versus standard insulin pump therapy in patients with Type 1 diabetes in Sweden. Diabetes Ther. 2017;8 (5):1015-1030. doi:10.1007/s13300-017-0294-z

41. Kasteng F, Eriksson J, Sennfalt K, Lindgren P. Metabolic effects and cost-effectiveness of aripiprazole versus olanzapine in schizophrenia and bipolar disorder. Acta Psychiatr Scand. 2011;124 (3):214-225. doi:10.1111/j.1600-0447.2011.01716.x

42. Kiadaliri AA, Gerdtham UG, Eliasson B, Carlsson KS. Cost-utility analysis of glucagon-like Peptide-1 agonists compared with dipeptidyl peptidase-4 inhibitors or neutral protamine hagedorn Basal insulin as add-on to metformin in type 2 diabetes in sweden. Diabetes Ther. 2014;5(2):591-607. doi:10.1007/s13300-014-0080-0

43. Kolu P, Raitanen J, Puhkala J, Tuominen P, Husu P, Luoto R. Effectiveness and cost-effectiveness of a cluster-randomized prenatal lifestyle Counseling Trial: a seven-year follow-up. PLoS One. 2016;11(12):e0167759. doi:10.1371/journal. pone.0167759

44. Landstedt-Hallin L, Gundgaard J, Ericsson A, Ellfors-Zetterlund S. Cost-effectiveness of switching to insulin degludec from other basal insulins: evidence from Swedish real-world data. Curr Med Res Opin. 2017;33(4):647-655. doi:10.1080/ 03007995.2016.1277194

45. Lindgren P, Lindström J, Tuomilehto J, et al. Lifestyle intervention to prevent diabetes in men and women with impaired glucose tolerance is cost-effective. Int J Technol Assess Health Care. 2007;23(2):177-183. doi:10.1017/S0266462307070286

46. Oostdam N, Bosmans J, Wouters MGAJ, Eekhoff EMW, van Mechelen W, van Poppel MNM. Cost-effectiveness of an exercise program during pregnancy to prevent gestational diabetes: results of an economic evaluation alongside a randomised controlled trial. BMC Pregnancy Childbirth. 2012;12(1). doi:10.1186/14712393-12-64
47. Patel A, Maissi E, Chang HC, et al. Motivational enhancement therapy with and without cognitive behaviour therapy for Type 1 diabetes: economic evaluation from a randomized controlled trial. Diabet Med. 2011;28(4):470-479. doi:10.1111/j.14645491.2010.03198.x

48. Roussel R, Martinez L, Vandebrouck T, et al. Evaluation of the long-term cost-effectiveness of liraglutide therapy for patients with type 2 diabetes in France. $J$ Med Econ. 2016;19 (2):121-134. doi:10.3111/13696998.2015.1100998

49. Roze S, de Portu S, Smith-Palmer J, Delbaere A, Valentine W, Ridderstrale M. Cost-effectiveness of sensor-augmented pump therapy versus standard insulin pump therapy in patients with type 1 diabetes in Denmark. Diabetes Res Clin Pract. 2017;128:6-14. doi:10.1016/j.diabres.2017.02.009

50. Roze S, Duteil E, Smith-Palmer J, et al. Cost-effectiveness of continuous subcutaneous insulin infusion in people with type 2 diabetes in the Netherlands. J Med Econ. 2016;19(8):742-749. doi:10.3111/13696998.2016.1167695

51. Roze S, Saunders R, Brandt AS, de Portu S, Papo NL, Jendle J. Health-economic analysis of real-time continuous glucose monitoring in people with Type 1 diabetes. Diabet Med. 2015;32 (5):618-626. doi:10.1111/dme.12661

52. Roze S, Smith-Palmer J, Valentine W, et al. Cost-effectiveness of sensor-augmented pump therapy with low glucose suspend versus standard insulin pump therapy in two different patient populations with type 1 diabetes in France. Diabetes Technol Ther. 2016;18 (2):75-84. doi:10.1089/dia.2015.0224

53. Roze S, Smith-Palmer J, Valentine WJ, et al. Long-term health economic benefits of sensor-augmented pump therapy vs continuous subcutaneous insulin infusion alone in type 1 diabetes: a U. K. perspective. J Med Econ. 2016;19(3):236-242. doi:10.3111/ 13696998.2015.1113979

54. Saha S, Carlsson KS, Gerdtham UG, et al. Are lifestyle interventions in primary care cost-effective?-An analysis based on a Markov model, differences-in-differences approach and the Swedish Bjorknas study. PLoS One. 2013;8(11):e80672. doi:10.1371/journal.pone.0080672

55. Slangen R, Faber CG, Schaper NC, et al. A trial-based economic evaluation comparing spinal cord stimulation with best medical treatment in painful diabetic peripheral neuropathy. J Pain. 2017;18(4):405-414. doi:10.1016/j.jpain.2016.11.014

56. Smith-Palmer J, Fajardo-Montanana C, Pollock RF, Ericsson A, Valentine WJ. Long-term cost-effectiveness of insulin detemir versus NPH insulin in type 2 diabetes in Sweden. J Med Econ. 2012;15(5):977-986. doi:10.3111/13696998.2012.692340

57. Steen Carlsson K, Persson U. Cost-effectiveness of add-on treatments to metformin in a Swedish setting: liraglutide vs sulphonylurea or sitagplitin. $J$ Med Econ. 2014;17(9):658-669. doi:10.3111/13696998.2014.933110

58. Tsiachristas A, Cramm JM, Nieboer AP, Rutten-van Mölken MPMH. Changes in costs and effects after the implementation of disease management programs in the Netherlands: variability and determinants. Cost Eff Resour Alloc. 2014;12(1):17. doi:10.1186/1478-7547-12-17

59. Valentine WJ, Aagren M, Haglund M, Ericsson A, Gschwend MH. Evaluation of the long-term cost-effectiveness of insulin detemir compared with neutral protamine hagedorn insulin in patients with type 1 diabetes using a basal-bolus regimen in Sweden. Scand J Public Health. 2011;39(1):79-87. doi:10.1177/1403494810379290

60. Valentine WJ, Palmer AJ, Lammert M, Langer J, Brandle M. Evaluating the long-term cost-effectiveness of liraglutide versus exenatide BID in patients with type 2 diabetes who fail to improve with oral antidiabetic agents. Clin Ther. 2011;33 (11):1698-1712. doi:10.1016/j.clinthera.2011.09.022 
61. Brown GC, Brown MM, Turpcu A, Rajput Y. The cost-effectiveness of ranibizumab for the treatment of diabetic macular edema. Ophthalmology. 2015;122(7):1416-1425. doi:10.1016/j.ophtha.2015.03.032

62. Cutino A, Green K, Kendall R, Moore PT, Zachary C. Economic evaluation of a fluocinolone acetonide intravitreal implant for patients with DME based on the FAME study. Am J Manag Care. 2015;21(4 Suppl):S63-S72.

63. Eddy DM, Kahn R, Schlessinger L. Clinical outcomes and cost-effectiveness of strategies for managing people at high risk for diabetes. Ann Intern Med. 2005;143(4):251-264. doi:10.7326/ 0003-4819-143-4-200508160-00006

64. Greeley SAW, John PM, Winn AN, et al. The cost-effectiveness of personalized genetic medicine: the case of genetic testing in neonatal diabetes. Diabetes Care. 2011;34(3):622-627. doi: $10.2337 / \mathrm{dc} 10-1616$

65. The Diabetes Prevention Program Research Group. Within-trial cost-effectiveness of lifestyle intervention or metformin for the primary prevention of type 2 diabetes. Diabetes Care. 2003;26 (9):2518-2523. doi:10.2337/diacare.26.9.2518

66. Haig J, Barbeau M, Ferreira A. Cost-effectiveness of ranibizumab in the treatment of visual impairment due to diabetic macular edema. $J$ Med Econ. 2016;19(7):663-671. doi:10.3111/ 13696998.2016.1154566

67. Herman WH, Ackermann RT, Brandle $M$, et al. The cost-effectiveness of lifestyle modification or metformin in preventing type 2 diabetes in adults with impaired glucose tolerance. Ann Intern Med. 2005;142(5):323-332. doi:10.7326/0003-4819142-5-200503010-00007

68. Huang ES, O'Grady M, Basu A, et al. The cost-effectiveness of continuous glucose monitoring in type 1 diabetes. Diabetes Care. 2010;33(6):1269-1274. doi:10.2337/dc09-2042

69. Kamble S, Schulman KA, Reed SD. Cost-effectiveness of sensor-augmented pump therapy in adults with type 1 diabetes in the United States. Value Health. 2012;15(5):632-638. doi:10.1016/j.jval.2012.02.011

70. Kuo S, Bryce CL, Zgibor JC, Wolf DL, Roberts MS, Smith KJ. Cost-effectiveness of implementing the chronic care model for diabetes care in a military population. J Diabetes Sci Technol. 2011;5(3):501-513. doi:10.1177/193229681100500305

71. Rosen AB, Hamel MB, Weinstein MC, Cutler DM, Fendrick AM, Vijan S. Cost-effectiveness of full medicare coverage of angiotensin-converting enzyme inhibitors for beneficiaries with diabetes. Ann Intern Med. 2005;143(2):89-99. doi:10.7326/00034819-143-2-200507190-00007

72. Valentine WJ, Palmer AJ, Erny-Albrecht KM, et al. Costeffectiveness of basal insulin from a US health system perspective_comparative analyses of detemir, glargine, and NPH. $A d v$ Ther. 2006;23(2):191-207. doi:10.1007/BF02850126

73. Farshchi A, Aghili R, Oskuee M, et al. Biphasic insulin Aspart 30 vs. NPH plus regular human insulin in type 2 diabetes patients; a cost-effectiveness study. BMC Endocr Disord. 2016;16(1):35. doi:10.1186/s12902-016-0116-8

74. Lian J, McGhee SM, Gangwani RA, et al. The impact of a co-payment on the cost-effectiveness of screening for diabetic retinopathy. $J$ Public Health (Oxf). 2016;38(4):782-792. doi:10.1093/pubmed/fdv168

75. Nguyen HV, Tan GS, Tapp RJ, et al. Cost-effectiveness of a National Telemedicine Diabetic Retinopathy Screening Program in Singapore. Ophthalmology. 2016;123 (12):2571-2580. doi:10.1016/j.ophtha.2016.08.021

76. Png ME, Yoong JS. Evaluating the cost-effectiveness of lifestyle modification versus metformin therapy for the prevention of diabetes in Singapore. PLoS One. 2014;9(9):e107225. doi:10.1371/journal.pone. 0107225
77. Agency NM. Guidelines on how to conduct pharmacoeconomic analyses. Oslo Norwegian Medicines Agency; 2012.

78. Capri S, Ceci A, Terranova L, Merlo F, Mantovani L. Guidelines for economic evaluations in Italy: recommendations from the Italian Group of Pharmacoeconomic Studies. Drug Inf J. 2001;35(1):189-201. doi:10.1177/009286150103500122

79. Kristensen FB, Sigmund H. Health Technology Assessment Handbook. Copenhagen: Danish Centre for Health Technology Assessment. National Board of Health; 2008.

80. López-Bastida J, Oliva J, Antonanzas F, et al. Spanish recommendations on economic evaluation of health technologies. Eur $J$ Health Econ. 2010;11(5):513-520. doi:10.1007/s10198-010-0244-4

81. Santé H Choices in methods for economic evaluation. Saint-Denis La Plaine: Haute Autorité de Santé; 2012.

82. Walter E, Zehetmayr S. [Guidelines for health-economic evaluations in Austria]. Wien Med Wochenschr. 2006;156(23-24):628632. German. doi:10.1007/s10354-006-0360-z

83. Zorginstituut N. [Richtlijn Voor Het Uitvoeren Van Economische Evaluaties in de Gezondheidszorg]. Diemen: Zorginstituut Nederland; 2015.

84. Drost RM, van der Putten IM, Ruwaard D, Evers SM, Paulus AT. Conceptualizations of the societal perspective within economic evaluations: a systematic review. Int J Technol Assess Health Care. 2017;33(2):251-260. Dutch. doi:10.1017/ S0266462317000526

85. Johannesson MJ, Jönsson B, Jönsson L, Kobelt G, Zethraeus N Why should economic evaluations of medical innovations have a societal perspective? OHE Briefing, No 51, Office of Health Economics, London; 2009.

86. Del Río-Lozano M, García-Calvente MDM, Marcos-Marcos J, Entrena-Durán F, Maroto-Navarro G. Gender identity in informal care: impact on health in Spanish caregivers. Qual Health Res. 2013;23(11):1506-1520. doi:10.1177/1049732313507144

87. Sharma N, Chakrabarti S, Grover S. Gender differences in caregiving among family - caregivers of people with mental illnesses. World J Psychiatry. 2016;6(1):7. doi:10.5498/wjp.v6.i1.7

88. Spijker J, Zueras P. Old-age care provision in Spain in the context of a new system of long-term care and a lingering economic crisis. J Popul Ageing. 2020;13(1):41-62. doi:10.1007/s12062018-9232-8

89. Commission E. The 2015 Ageing Report: Economic and Budgetary Projections for the 28 EU Member States (2013-2060). Luxembourg; 2015.

90. Commission E. The 2018 Ageing Report: Economic and Budgetary Projections for the EU Member States (2016-2070). Brussels; 2018.

91. Shafie AA, Ng CH, Tan YP, Chaiyakunapruk N. Systematic review of the cost effectiveness of insulin analogues in type 1 and type 2 diabetes mellitus. Pharmacoeconomics. 2017;35 (2):141-162. doi:10.1007/s40273-016-0456-2

92. Baptista A, Teixeira I, Romano S, Carneiro AV, Perelman J. The place of DPP-4 inhibitors in the treatment algorithm of diabetes type 2: a systematic review of cost-effectiveness studies. Eur J Health Econ. 2017;18(8):937-965. doi:10.1007/s10198-016-0837-7

93. Leichter S. Is the use of insulin analogues cost-effective? $A d v$ Ther. 2008;25(4):285-299. doi:10.1007/s12325-008-0043-9

94. Beaudet A, Palmer JL, Timlin L, et al. Cost-utility of exenatide once weekly compared with insulin glargine in patients with type 2 diabetes in the UK. $J$ Med Econ. 2011;14(3):357-366. doi:10.3111/13696998.2011.579213

95. Palmer JL, Goodall G, Nielsen S, et al. Cost-effectiveness of insulin aspart versus human soluble insulin in type 2 diabetes in four European countries: subgroup analyses from the PREDICTIVE study. Curr Med Res Opin. 2008;24 (5):1417-1428. doi:10.1185/030079908X297295 
96. Coyle KB, Trochlil K, Iversen P. MEDLINE and EMBASE for health economic literature reviews [abstract]. Value Health. 2012;15(4). doi:10.1016/j.jval.2012.03.877

97. McKinlay RJ, Wilczynski NL, Haynes RB; Team. tH. Optimal search strategies for detecting cost and economic studies in EMBASE. BMC Health Serv Res. 2006;6(1). doi:10.1186/14726963-6-67

98. Sassi F, Archard L, McDaid D. Searching literature databases for health care economic evaluations: how systematic can we afford to be? Med Care. 2002;40(5):387-394. doi:10.1097/00005650200205000-00004

99. Shemilt I, Mugford M, Vale L, Craig D. Searching NHS EED and HEED to Inform Development of Economic Commentary for Cochrane Intervention Reviews. Oxford:The Cochrane Collaboration; 2011.

100. Neumann PJ, Greenberg D, Olchanski NV, Stone PW, Rosen AB. Growth and quality of the cost-utility literature, 1976-2001. Value Health. 2005;8(1):3-9. doi:10.1111/j.1524-4733.2005.04010.x

101. Joo H, Zhang P, Wang G. Cost of informal care for patients with cardiovascular disease or diabetes: current evidence and research challenges. Qual Life Res. 2017;26(6):1379-1386. doi:10.1007/ s11136-016-1478-0
102. Li TC, Lee YD, Lin CC, Amidon RL. Quality of life of primary caregivers of elderly with cerebrovascular disease or diabetes hospitalized for acute care: assessment of well-being and functioning using the SF-36 health questionnaire. Qual Life Res. 2004;13(6):1081-1088. doi:10.1023/B:QURE.0000031349.25124.d6

103. Stedman M, Lunt M, Davies M, et al. Cost of hospital treatment of type 1 diabetes (T1DM) and type 2 diabetes (T2DM) compared to the non-diabetes population: a detailed economic evaluation. BMJ Open. 2020;10(5):e033231. doi:10.1136/bmjopen-2019033231

104. Association AD. 2. Classification and diagnosis of diabetes. Diabetes Care. 2017;40(Suppl. 1):S11-S24. doi:10.2337/dc17S005

105. National Institute of Diabetes and Digestive Kidney Diseases N. Your Guide to Diabetes: Type 1 and Type 2. NIH Publication; 2013:14.

106. da Rocha Fernandes J, Ogurtsova K, Linnenkamp U, et al. IDF diabetes atlas estimates of 2014 global health expenditures on diabetes. Diabetes Res Clin Pract. 2016;117:48-54. doi:10.1016/ j.diabres.2016.04.016
ClinicoEconomics and Outcomes Research

\section{Publish your work in this journal}

ClinicoEconomics and Outcomes Research is an international, peerreviewed open-access journal focusing on Health Technology Assessment, Pharmacoeconomics and Outcomes Research in the areas of diagnosis, medical devices, and clinical, surgical and pharmacological intervention. The economic impact of health policy and health systems

\section{Dovepress}

organization also constitute important areas of coverage. The manuscript management system is completely online and includes a very quick and fair peer-review system, which is all easy to use. Visit http://www.dovepress.com/testimonials.php to read real quotes from published authors. 